\title{
Ueber das mikroskopische Verhalten des Myometriums bei pathologischen Vergrösserungen des Uterus mit besonderer Beriicksichtigung der Iuskelzellen.
}

\author{
Von
}

Richard Bertelsmann,

Volontärassistent an pathologischen Institut zn Göttingen.

Die Vergrösserungen, welche der menschliche Uterus bei verschiedenen krankhaften Veränderungen erleidet, sind so in die Augen springend und durch ihre diagnostische Verwerthbarkeit so in den Vordergrund gestellt, dass sie stets das Interesse der Gynäkologen und Pathologen erregt haben. Man findet deshalb in der Literatur Aeusserungen vieler Autoren über diesen Gegenstand. Genauere Angaben über das histologische Verhalten der hypertrophirten Uteruswand sind aber meines Wissens nur von zwei Autoren gemacht worden. Man beschränkt sich darauf festzustellen, ob bei dieser oder jener Art der Uterushyperthrophie die Vergrösserung auf Rechnung der Muskulatur oder auf die des Bindegewebes kommt. Ueber das macroscopische Verhalten haben wir aus älterer und neuerer Zeit mancherlei theilweise sehr genaue Angaben. Besonders sind die Fälle erwähnt, wo der hypertrophische Uterus dem schwangeren ähnlich sah.

So widmet Virchow in seinen "Krankhaften Geschwülsten" dem Gegenstande eine eingehende Besprechung, soweit die Uterushypertrophie durch Fibro-Myome herbeigeführt wird. Dieselbe kommt nach ihm bei interstitiellen und submucösen Fibro-Myomen vor, ist aber weder eine nothwendige Bedingung der Tumorbildung, noch zieht die Tumorbildung immer Hypertrophie nach sich. Es kann nach ihm auch Atrophie der Uteruswand bei Myombildung bestehen, doch ist diese Atrophie möglicherweise eine secundäre, erst im späteren Alter sich einstellende. Auch ist diese Atrophie nach Virchow sehr selten. Fast immer ist vielmehr bei submu- 
cösem Fibro-Hyom die Wand bis zu $2-3 \mathrm{~cm}$ verdickt. Ihr Aussehen sowohl als ihre Zusammensetzung gleicht in hohem Maasse der des schwangeren Uterus. Man sieht die Fleischbalken stark entwickelt. Die Gefässe besonders die Venen weit und die Arterien starkwandig; selten ist die Wand des Uterus hart, meist hat sie die weichere, wirklich fleischige Beschaffenheit des schwangeren Uterus.

Ungefähr dasselbe fand Virchow bei den interstitiellen FibroMyomen. Andere ältere Autoren sind bald geneigt der Uterusvergrösserung durch Bindegewebe eine grössere Bedentung zuzusprechen, bald schliessen sie sich Virchow an. Im Allgemeinen sind ihre Ansichten folgende:

Eine Uterusvergrösserung, welche mehr auf einer Vergrösserung der Bindegewebsmasse beruht, findet bei entzündlichen Vorgängen in der Uteruswand, bei Stauungen, bei Lageveränderungen und bei sexuellen Excessen statt.

Ist das Uteruscavum durch Fibro-Myome, Blut, Schleim u. s. w. ausgefüllt und erweitert, dann tritt die Uterusvergrösserung ein, welche den Uterus dem schwangeren ähnlich macht und auf Zunahme der muskulären Elemente beruht.

Es äussern sich in dieser Weise Wenzel (Krankheiten des Uterus, Mainz 1816), Cruveilhier (in seinem Atlas der pathologischen Anatomie, Paris 1835), Foerster (Lehrbuch der pathologischen Anatomie, Leipzig 1855), Kiwisch (Klinische Vorträge über specielle Pathologie und Therapie der Krankheiten des weibliehen Geschlechts, Prag 1849), Scanzoni (Krankheiten der weiblichen Sexualorgane, Wien 1857), Oppolzer (Wiener medicinische Wochenschrift, Jahrgang 1858, p. 328), Rokitansky (Pathologische Anatomie, Wien 1861), Cornil und Ranvier (Pathologische Anatomie), Säxinger (Krankheiten des Uterus, Prager Vierteljahrsschrift für practische Heilkunde, Band 89, Jahrgang 1866), Gyon (Thèse de Paris, 1860. Des tumeurs fibreuses de l'utérus.) Birch-Hirschfeld (Lehrbuch der pathologischen Anatomie, Leipzig 1877).

Nirgends aber findet man ein genaueres Eingehen auf die Grösse der einzelnen Gewebselemente. Nur Klob (Pathologische Anatomie der weiblichen Sexualorgane, Wien 1864) stellt fest, dass, wenn man von einer echten Hypertrophie der Uteruswand sprechen wollte, die einzelnen Muskelzellen vergrössert sein müssten, und dass bei echter Hyperplasie der Uteruswand die einzelnen Gewebs- 
elemente so vermehrt sein müssten, dass sie in gleichen Verhältnissen zu einander bleiben. Klob hält derartige Zustände für sehr selten und geht nicht weiter auf das Thema ein. Von neueren Gynäkologen waren mir die Lehrbücber von Schröder (Leipzig 1890), Braun yon Fernwald (Wien 1880), Samuel Pozzi (Basel 1892), Fritsch (Frauenkrankheiten, Berlin 1892), Fehling (Wien 1893), Curty (Traité de maladies de l'utérus, Paris 1881) zur Hand. Die Anschauungen dieser Autoren, welche sich nicht wesentlich von den oben angeführten unterscheiden, sind im Allgemeinen folgende: Bei chronischer Metritis (chronischem Infarkt) beruht die Vergrösserung des Uterus auf Bindegewebswucherung, bei abnormem Inhalt der Uterushöhle tritt Vergrösserung und Umbildung der Uteruswände, ähnlich wie beim schwangeren Uterus, ein, doch ist auch Verdünnung der Wände des dann blasenförmig aufgetriebenen. Uterus nicht selten. Subseröse Fibro-Myome verursachen Zerrungen der Gebärmutter, interstitielle und submucöse Vergrösserungen, welche den Vergrösserungen während der Schwangerschaft häufig sehr ähnlich sind. Bei interstitiellen Fibro-Myomen ist die Vergrösserung aber bald unregelmässig, bald beruht sie nicht auf wahrer Hypertrophie sämmtlicher Gewebselemente, sondern auf diffuser Bindegewebswucherung. Von einer nachgewiesenen Vergrösserung der Muskelfaserzellen des Uterus ist nirgends die Rede. Speciell mit den Uterusvergrösserungen beschäftigte - sich in neuerer Zeit Ch. Labbé (in den Archives générales de Médicine, 1885).

An 10 Fällen beweist er, dass es eine totale idiopathische Hypertrophie des Uterus giebt. Er unterscheidet eine fibro-myomatöse und eine myo-teleangiektatische Hypertrophie. Bei der ersteren sind die Muskelzellen mässig vermehrt, das Bindegewebe viel weniger entwickelt. Das letztere ist regellos unter die Muskelzellen zerstreut, mit ihnen verfilzt. Bei der letzteren wird der Uterus zum cavernösen Gewebe.

Bei dieser Art der Uterusvergrösserung kann sich auch secundäre Cysten- und Tumorenbildung einstellen. Als solche idiopathische Hypertrophie mit secundärer oder accidenteller Bildung von kleinen Fibro-Myomen fasst Labbé auch einen von Tillaux der Societé de chirurgie demonstrirten Fall auf. (Gazette des hôpitaux, 1868, Nr. 144 und 146).

Bei einem meiner unten angeführten Fälle scheint mir, beiläufig gesagt, die gleiche Erklärung zulässig. Derselbe Autor führt über 
Uterusvergrösserungen noch die Ansicht de Sinéty's an, dessen Werk mir leider nicht zugänglich war. Derselbe scheint sich den unten angeführten Ansichten Ziegler's zu nähern, aber auch keine Angaben über Grössenverhältnisse der Muskelzellen zu machen.

Ziegler nun geht in seinem Lehrbuch der pathologischen Anatomie (1890) etwas näher auf die histologischen Verhältnisse bei den nicht in Folge von Gesehwülsten oder abnormem Inhalt entstandenen Vergrösserungen des Uterus ein. Ich führe seine Aeusserungen wörtlich an, weil sich meine Befunde ziemlich mit ihnen decken. Nach ihm findet man bei den Vergrösserungen, welche sich an das Puerperium anschliessen, also auf mangelhafter Involution beruhen, in einem Theil der Fälle Zunahme der Muskelmasse, ohne dass sich - wenigstens in späterer Zeit - entzündliche Veränderungen nachweisen liessen. In anderen Fällen sieht man, wenn die Individuen zur Section kamen, ehe der Entzündungsprocess abgelaufen ist, welcher sich in diesen Fällen nach der Entbindung eingestellt hatte, das Gewebe von zahlreichen in der Nähe der Gefässe und in der Nähe von grösseren Bindegewebssträngen gelegenen Rundstellen durchsetzt. Später entwickelt sich hier eine Zunahme der Bindegewebsmasse. Doch vergrössern sich die Muskelbündel ebenfalls. Zuweilen ist auch das innerhalb der Muskelzüge gelegene Bindegewebe verbreitert. Aehnliche Zustände sollen sich nach den übrigen Ursachen der Uterusvergrösserung: Endometritis, chronische Stauungen u.s. w. einstellen.

Eine weitere Aehnlichkeit zwischen dem Verhalten der Wand des schwangeren Uterus und der bei Anwesenheit von Fibro-Myomen verdickten fand Carl Ruge (Ueber die Contraction des Uterus in anatomischer und klinischer Beziehung. Zeitschrift für Geburtshülfe und Gynäkologie, Jahrgang 1880, Band 5).

Derselbe stellt fest, dass die Wand des schwangeren Uterus eine dem Peritoneum parallele Schichtung zeige; die. Schichten sind durch Verbindungsbrücken von Uterusgewebe verbunden. Hört die Schwangerschaft auf, so verschwindet die Schichtung bald, auf hier nicht zu erörternde Weise. Dieselbe lamellöse Schichtung sah Ruge auch, wenn Fibro-Myome das Uteruscavum ausdehnten. Bei meinen Untersuchungen fanden sich diese Schichtungen häufig und jedesmal mit ihnen verbunden eine Vergrösserung der Muskelzellen.

Die einzige Angabe über die Grösse der Muskelfaserzellen aus älterer Zeit, welche ich erst an dieser Stelle des Zusammen- 
hangs wegen einfüge, macht Dr. W. Finn, „Ueber die Veränderungen des Muskel- und Bindegewebes bei chronischer Metritis" im Centralblatt für die medicinischen Wissenschaften, Jahrgang 1868, Nr. 36 in einer vorläufigen Mittheilung, welche leider nur eine vorläufige geblieben zu sein scheint, denn es war mir nicht möglich eine zweite Veröffentlichung desselben Autors in der Literatur aufzufinden. In djeser werden auf Grund von Zerzupfungspräparaten folgende Resultate angegeben:

1. In der chronisch entzündeten Gebärmutter wird die regelmässige Lagerung sowohl der einzelnen Muskelfasern als auch der Bündel derselben unverändert erhalten.

2. Die Muskelfasern verändern sich nicht in qualitativer Beziehung und die fettige Entartung derselben stellt keine wesentliche constante Erscheinung bei der bezeichneten Krankheit vor.

3. Die Muskelfasern sind immer ausgedehnt und zwar mehr in die Länge als in die Breite.

4. Die Zahl der Fasern ist constant und bedeutend vermehrt.

5. Die Menge des Bindegewebes im späteren Stadium der Krankheit ist stets relativ vermindert, absolut aber. vermehrt, so dass die Zunahme des Volums der Gebärmutter durch die Hyperplasie der Muskelfasern bedingt wird und die Zunalime des Bindegewebes dabei einen sehr geringen Einfluss hat.

In neverer Zeit schreibt Herr Professor Orth, mein hochverehrter Lehrer, welchem ich auch an dieser Stelle für die freundliche Anregung und Anleitung zu der vorliegenden Arbeit danke, über die pathologischen Uterusvergrösserungen folgendes: (Lehrbuch der pathologischen Anatomie. II. Band.) Es giebt muskuläre Hypertrophien und bindegewebige. Jene wird man vorzugsweise erwarten dürfen, wo eine Wirkung vermehrter Arbeit oder der immanenten Anlage vorliegt, diese sind vorzugsweise in den Fällen vorhanden, wo entzündliche Vorgänge mitgewirkt haben. Die hypertrophische Muskulatur kann in Bezug auf Grösse der Muskelzellen den gewöhnlichen Befund des ruhenden Uterus geben, ich habe aber auch bei Myomen Muskelzellen gesehen, welche in ihren Dimensionen jener des schwangeren Uterus kaum nachstanden.

Her Professor Orth hatte nun die Güte, mich mit der Untersuchung der genauen Grössenverhältnisse der Muskelzellen in vergrösserten Gebärmüttern zu beauftragen.

Es wurde von mir die Wand von 28 Gebärmättern untersucht. 
Unter diesen befanden sich zwei mit an Endometritis erkrankter Schleimhaut und metritisch veränderten Wandungen. In die Fundushöhle des einen ragte ein kleiner Schleimpolyp ein, dem Fundus des anderen sass ein haselnussgrosses subseröses Fibro-Myom auf. Fin dritter Uterus war nur an Endometritis und Metritis erkranki, 4 Gebärmütter trugen Cervixcarcinome, 6 einfache, 3 multiple interstitielle und 5 submucöse Fibro-Myome. Unter den Gebärmüttern mit einfachen interstitiellen Myomen ist bei Nr. 14 ein Uterus aufgeführt, der ausserdem noch ein submucöses Fibro-Myom, bei Nr. 18 ein Uterus, der noch mehrere kleine Fibro-Myome trug. Es überwogen in diesen Fällen die interstitiellen Fibro-Myome so bedeutend an Grösse über die anderen und die letzteren waren im Verhältniss zur Uteruswand so klein, dass durch sie gewiss kein Einfluss auf die Vergrösserung des Uterus ausgeübt werden konnte. Dasselbe findet mutatis mutandis bei Nr. 20, 21 und 23 statt, welche unter den submucösen Myomen aufgeführt sind, obwohl sich noch ein oder mehrere kleine und bedeutungslose Fibro-Myome in den unter diesen Zahlen beschriebenen Organen befinden. Ein weiterer Uterus enthielt eine zuletzt in Eiter übergegangene Blutansammlung (Hämatometra resp. Pyometra), ein anderer ein in die Uterushöhle hineinragendes Deciduom, ein anderer ein eben solches Sareom.

Als Vergleichsobjecte wurden ausserdem noch die Uteri dreier an Febris puerperalis gestorbener Frauen und ein ausgebildeter jungfräulicher Uterus untersucht.

Die Stücke zur microscopischen Untersuchung wurden möglichst immer aus der gleichen Gegend des Organs, nämlich aus der Vitte zwisehen dem Scheitel des Fundus und dem inneren Muttermunde entnommen. An den frisch in meine Hände kommenden Organen wurden Messungen der Muskelzellen an Zerzupfungspräparaten gemacht, welche mit 33 pCt. Kalilauge hergestellt waren. Aeltere in Alkohol aufbewahrte Gebärmütter wurden mitersucht, indem möglichst kleine Stücke derselben 1-2 Tage in 30 proc. Salpetersäure gelegt und dann in derselben zerzupft wurden. Es liessen sich auf diese Weise in den meisten Fällen die Muskelfaserzellen auch recht alter Präparate noch ganz gut isoliren.

Allerdings sahen die Muskelzellen eigenthümlich gewellt aus, auch sah ich häufig stärkere Körnungen im Zellleib, doch hatte ich bei Vergleichen mit den frisch zerzupften Präparaten den Eindruck, als seien die Grössenverhältnisse nicht wesentlich ver- 
ändert. Der gewellte Verlauf der Muskelzellen hätte die Längemessungen hauptsächlich beeinträchtigen können, es zeigten sich aber -- wie man unten sehen wird -- so bedeutende Unterschiede, dass es auf einige Micromillimeter auf oder ab für meine Zwecke nicht ankam. Schnittpräparate von frischen Organen wurden in den Härtungsflüssigkeiten immer gleiche Zeiträume hindurch belassen, um sie nicht verschieden starke Schrumpfung veranlassenden Einflüssen auszusetzen.

Bei den älteren Präparaten war eine derartige Vorsicht nicht mehr möglich, da ich aber hauptsächlich solche alte Alkoholpräparate untersuchte, in denen Vergrösserungen der Muskelzellen festzustellen waren, so können die Resultate bei diesen nicht angezweifelt werden, man kann vielmehr sagen, dass mindestens diese Vergrösserungen vorhanden waren.

Vorgehärtet wurden die frischen Organe in Müller'scher Flüssigkeit oder Formalin, zuerst in den genannten Flüssigkeiten je ein Stück des gleichen Falls. Es zeigten sich hierbei keine Verschiedenheiten in den Querschnittsmaassen der Muskelzellen. Die älteren Präparate waren grossentheils in Müller'scher Flüssigkeit vorgehärtet, alle in Alkohol aufbewahrt. Sämmtliche Präparate wurden in steigendem Alkohol nachgehärtet und in Paraffin eingebettet.

Gefärbt wurde mit Methylenblau-Eosin- und Pikrolithioncarmin. Mit der letzteren Farbe erhielt ich die schönsten Differenzirungen zwischen Muskulatur und Bindegewebe. Die erstere wird gelb mit rothem Kerne, das letztere blassroth mit rothem Kerne. Bei stärkerer Vergrösserung sieht ausgebildetes Bindegewebe bei dieser Färbung weiss mit leicht röthlichem Schimmer aus. Die Präparate, an welchen ich Querschnittsmessungen an Muskelzellen machen wollte, brachte ich, nachdem sie in Salzsäurealkohol wie die anderen differenzirt waren, für 3-5 Minuten in concentrirte alkoholische Pikrinsäurelösung.

Hierdurch geht zwar in manchen Fällen der deutliche Farbenunterschied zwischen Muskulatur und Bindegewebe verloren, indem das letztere auch einen gelben Farbenton annimmt, aber die Muskelzellen färben sich so intensiv, dass man ihre Grenzen deutlich erkennen und ihre Dimensionen in den meisten Fällen leicht auch an Schnittpräparaten messen kann. Die Untersuchung und Messung auch an Schnittpräparaten vorzunehmen halte ich für werthroll, da man einerseits sich nur so über all- 
gemeine Verhältnisse, die Menge und Art des Bindegewebes; das Verhalten der Gefässe u. s. w. klar werden kann; andererseits man sehen kann, ob es sich um allgemeine Hypertrophien handelt, oder ob sich nur hier und da ein einzelnes hypertrophisches Muskelbündelchen findet, was, da die Histogenese der Fibro-Myome noch nicht aufgeklärt ist, ebenso gut Anfang eines Myoms wie der Anfang einer wahren muskulären Hypertrophie sein kann. Bei den Zerzupfungen liegt auch in meinen Fällen die Gefahr vor, ein kleinstes Fibro-Myomknötchen zu zerzupfen, welches, obwohl schon eine ausgebildete Geschwulst, sich dem blossen Auge entzieht und, wie ich nebenbei sah, kamen in Fibro-Myomen kolossale Muskelzellen vor. Diese Gefahr wird durch die Combination von Zerzupfungspräparaten mit Schnittpräparaten vermieden. Bei den Messungen an Querschnitten ging ich von der Ueberlegung aus, dass auch, wenn man reine Querschnitte erhalten hat, nicht sämmtliche Muskelzellen in ihrer Mitte, also, da sie spindelförmige Gebilde sind, an ihrem grössten Umfang getroffen sein könnten, dass vielmehr jedes Muskelbündelchen, da die Muskelzellen keineswegs alle in gleicher Höhe liegen, Muskelzellquerschnitte zeigen wird, deren Dimensionen sich von den grössten bis zu den kleinsten abstufen, je nachdem die Muskelzellen in der Mitte oder an einem der Enden getroffen sind. Tiegt nun eine wahre Hypertrophie der Muskelzellen vor, so müssen sich in vielen Muskelbündelchen den normalen gegenüber vergrösserte Muskelzell-Querschnitte zeigen. Meistens wird jedes Muskelbündelchen mehrere solche vergrösserte Querschnitte haben, manchmal sind auch fast alle vergrössert, dies wird stattfinden, wenn die Muskelzellen sehr lang sind, und, was in diesem Falle häufig ist, in der Mitte lange Zeit parallel verlaufende Grenzen haben. Findet man in einem Schnitt, der sonst bedeutende Querschnittsvergrösserungen enthält, ein oder mehrere Muskelbündelchen, welche keine Vergrösserungen aber viele Muskelzellen ohne Kerne zeigten, so wird man ein Muskelbündel an seinem Ende durch den Schnitt getroffen haben und dieser Befund ist kein Gegenbeweis gegen eine allgemeine Hypertrophie der Muskelzellen. Um zu verhüten, dass Schrägschnitte anstatt Querschnitte gemessen und so unrichtige Zahlen erzielt würden, maass ich nur solche Muskelzellen, deren Kerne ohne sich seitlich zu verschieben - zu wandern - bei der Drehung der Micrometerschraube versehwanden. Ein Hülfscriterium um hyper- 
trophische Degenerations- und Wucherungsvorgänge zu erkennen, ist die Zahl und die Gestalt der Kerne.

Dieselben werden bei kleinen Muskelzellen in grosser Anzahl dicht bei einander liegen, was man am ersten bei längsgetroffenen Muskelbündelchen sehen kann; handelt es sich um lebenskräftige Muskelzellen, so wird man nach Stilling und Pfitzner's Untersuchungen in ihnen ein Chromatingerüst sehen, dessen einzelne sehr feine Fäden man deutlich unterscheiden kann. Kerne kurz vor der Theilung werden ein ebenfalls deutliches Chromatingerüst zeigen mit feinen Fäden, aber sehr lang sein. Liegt eine Neubildung von Muskelzellen vor, so sieht man zwischen langen Kernen, kurze dicht neben einander liegende im Verhältniss zur Länge breite Kerne. Kerne in ihrer Ernährung gestörter vergrösserter Muskelzellen sind lang, schmal, bei Methylenblaufärbung lässt sich überhaupt kein Chromatingerüst, bei Pikrolithioncarminfärbung ein solches nur sehr schwer erkennen. Dasselbe ist "verklumpt". Die Kerne geschwächter und nicht hypertrophischer Muskelzellen sind kurz, schmal und lassen kein deutliches Chromatingerüst erkennen. Da es sich nicht um gleich frisches Material handelte sind Schlüsse aus dem Verhalten des Chromatins in den Kernen nur mit Vorsicht zu machen.

Von diesen Gesichtspunkten aus lassen sich die Verhältnisse der Gewebselemente in der Uteruswand gut beurtheilen. Für Dickenmessungen an den Muskelzellen war die Untersuchung an Zerzupfungspräparaten zur Controle sehr erwünscht, für Längenmessungen unerlässlich. Die Länge und Breite wurden immer für je eine Muskelzelle festgestellt, um das Verbältniss der beiden zu erhalten. Die Zerzupfungspräparate wurden so hergestellt, dass ganz kleine Theilchen, eins aus der Nähe der Serosa, eins aus der Nähe der Mucosa und eins aus der Mitte der Uteruswand entnommen wurden. Diese wurden zerzupft und das Zerzupfte gemischt. Es wurden dann in den zufällig eingestellten Gesichtsfeldern alle gut isolirten Muskelzellen gemessen, um Maasse aller vorkommenden Grössen zu erhalten. Bei den Organen, welche Carcinome enthielten, wurde von dieser Regel abgewichen, da dort kleinere Muskelzellen in den Gesichtsfeldern in einer Anzahl erschienen, welche dem Befund an Schnittpräparaten nicht entsprach. Es wurden hier die grössten und die kleinsten Muskelzellen - von jeder Art sechs - gemessen und hieraus der Durchschnitt gezogen. 
Angaben aus den Krankengeschichten sind nur gemacht worden, wenn sie von Belang erschienen. Gravidität wurde natürlich stets ausgeschlossen.

1. Uterus von einer 40 jährigen, an Pneumonie gestorbenen Frau:

Das vorliegende Organ ist mässig vergrössert, seine Wände sind in der Mitte zwischen innerem Muttermunde und dem Scheitel des Fundus gemessen $1^{1 /} / 4 \mathrm{~cm}$ dick, die Farbe ist auf dem Durchschnitt grauweiss, die Consistenz fest.

Die Schleimhaut des Uterus ist endometritisch verändert, in der Fundushöhle befindet sich ein kleinbohnengrosser Schleimpolyp.

Die mikroskopische Untersuchung ergiebt, dass reichliches, zellarmes, ausgebildetes Bindegewebe, welches besonders in der Nähe der Gefässe in breiten Zügen hervortritt, die Muskelbündelchen umgiebt und sich auch im Innern derselben stark entwickelt hat. Hier und da, besonders in der Nähe der Schleimbaut, sieht man kleine Rundzellen in kleinen Häufchen.

In den Muskelbündelchen sieht man sehr viele kurze Kerne, welche ein undeutliches Chromatingerüst reigen und, wo die Yuskelzellen nicht sichtlich durch Bindegewebe auseinandergedrängt sind, eng stehen. Vereinzelt sieht man auch Muskelbündelchen, in welchen etwas längere Kerne weiter von einander entfernt liegen.

Messungen an Querschnitten waren sehr schwer zu machen, doch komnte festgestellt werden, dass der Durchschnitt von 6 Querschnittsmessungen $4,4 \mu$ betrug. An Zerzupfungspräparaten erhielt ich folgende Werthe:

\begin{tabular}{|c|c|c|c|c|c|c|}
\hline inge & 39 & $34 \frac{2}{3}$ & 43 & 39 & 83 & $55^{1 / 3}$ \\
\hline re & $6^{1 / 3}$ & $41 / 3$ & 5 & 4 & $4^{1} / 3$ & \\
\hline ä̀n & 39 & 26 & 30 & $34^{2} / 3$ & $301 / 3$ & 43 \\
\hline Breite & 3 & 3,9 & 2,6 & 3,1 & $41 / 3$ & \\
\hline \multicolumn{2}{|c|}{ Durchschnittlich } & Länge & $41^{5} / 12$ & & Breite & \\
\hline \multicolumn{2}{|c|}{ Höchste Zahl } & & 83 & & & \\
\hline \multicolumn{2}{|c|}{ Niedrigste Zahl } & $"$ & 26 & & & \\
\hline \multicolumn{2}{|c|}{ Differenz } & $\eta$ & 57 & & $m$ & 3,7 \\
\hline
\end{tabular}

2. Uterus von einer 60 jährigen, an Mamma-Carcinommetastasen gestorbenen Frau.

Dieser Uterus ist ebenfalls etwas vergrössert; seine Länge beträgt $8 \mathrm{~cm}$, der grösste frontale Durchmesser am Fundus $4 \mathrm{~cm}$, die Dicke der Wand ist nicht vergrössert, sie beträgt nur $3 / 4 \mathrm{~cm}$. Die $\mathrm{Zu}-$ nahme der äusseren Maasse muss also anf Erweiterung der Fundushöhle beruhen. Dem Fundus sitzt ein haselnussgrosses subseröses Fibromyom auf. Die Schleimhaut zeigt endometritische Veränderungen.

In der Fundushöhle inserirt sich oben in der Mittellinie ein Schleimpolyp von der Grösse des Nagelgliedes eines kleinen Fingers. Die Farbe ist auf dem Wunddurchschnitt grauweiss. Die Wand fühlt sich hart an.

Mit dem Mikroskop sieht man, dass das Bindegewebe sehr reichlich entwickelt und zellarm, faserig ist. Breite Züge mit spärlichen Rundzellen durchsetzt sieht man besonders in der Nähe der Gefässe. In den Muskelbündeln selbst ist keine reichlichere Bindegewebsentwickelung eingetreten, diese selbst sind schmal. Die Kerne liegen in ihnen dicht 
bei einander, sind kurz und schmal und lassen ein Chromatingerüst nur undeutlich erkennen. Messungen an Muskelzellquerschnitten waren wegen der Kleinheit und dichten Aneinanderlagerung der Muskelzellen sehr schwierig und ergaben kein sicheres Resultat. An Zerzupfungspräparaten ergaben sich folgende Zahlen:

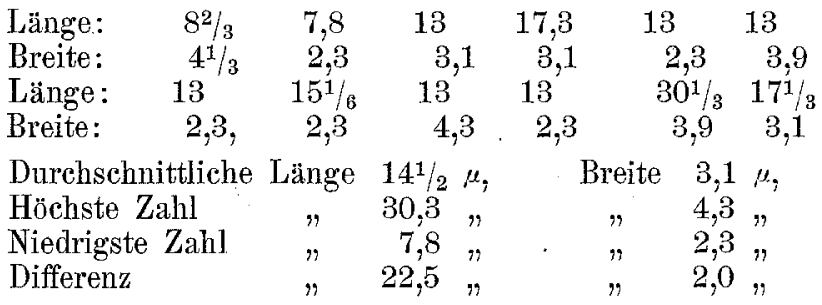

3. Uterus von einer 42 jährigen Frau, welche seit einem Jahre an Endometritis behandelt wurde. Das Organ wurde durch Operation entfernt.

Die Länge dieses Uterus beträgt $10 \mathrm{~cm}$; die Dicke der mittelfesten Wand $2 \mathrm{~cm}$; die Breite des Organs am Fundus $6 \mathrm{~cm}$. An der Schleimhaut wird eine proliferirende Endometritis festgestellt. Die Farbe der Wände ist auf dem Durchschnitt grauröthlich; das ganze Organ ziemlich blut- und saftreich. Schichtungen sind an der Wand nicht zu erkennen.

Zunächst der Schleimhaut sieht man vorwiegend Bindegewebe, welches bier und da Züge, welche nach der Schleimhaut zu vordringen und weit in sie hineinreichen, entsendet und hier und da entzündlich infiltrirt ist. Zwischen diesem, näher der Serosa zu, treten Bündelchen von 2-3-4 Muskelzellen auf, welche, je mehr man der Serosa nahe kommt, desto zahlreicher und umfangreicher werden, das Bindegewebe ist zum Theil sehr zellreich, hauptsächlich aber in der Schleimhautnähe, wo es oft nur schwer vom ovalzelligen Stroma der Schleimhaut unterschieden werden kann, mehr der Serosa zu wird es zellärmer, faseriger und umzieht die Muskelbündel in breiten, weisslich-röthlich glänzenden Zügen. Auffällig ist hier, wie die grösseren Muskelbündel von Bindegewebe durchflochten sind. Dieselben täuschen oft bei schwacher Vergrösserung eine rein muskuläre Hyperplasie vor. Bei starker Vergrösserung sieht man dann bei Pikrolithionkarmin-Färbung besonders gut, dass die einzelnen Muskelzellen von verbreitertem weiss-röthlichem Bindegewebe umgeben sind, so dass es den Eindruck macht, als seien viele kleine Rärume für die Muskelzellen in der Bindegewebsmasse ausgespart. Was also anfänglich muskuläre Hyperplasie zu sein schien, erweist sich als theilweise Bindegewebshyperplasie.

Nahe der Serosa sind auch Muskelbündel, welche wenig Bindegewebe enthalten und in denselben Muskelzellen, welche 6-9,6 $\mu$ im Durchmesser haben, während in den übrigen Theilen des Schnitts sich nur Dimensionen unter oder bis $4 \mu$ zeigen. Die Kerne der Muskelzellen sind lang und breit, sie zeigen deutliches Chromatingerüst. Besonders in der Nähe der Serosa liegen sie ziemlich weit von einander entfernt. In Schnitten von Stücken, welche $1 / 2$ Stunde nach der Operation in Zenker'sche Fixationsflüssigkeit gelegt wurden, sieht man oft zwei kurze, breite Kerne so nahe zusammenliegen, dass man an eine 
eben abgelaufene Kerntheilung denken kann. An Zerzupfungspräparaten wurden folgende Grössen gemessen:

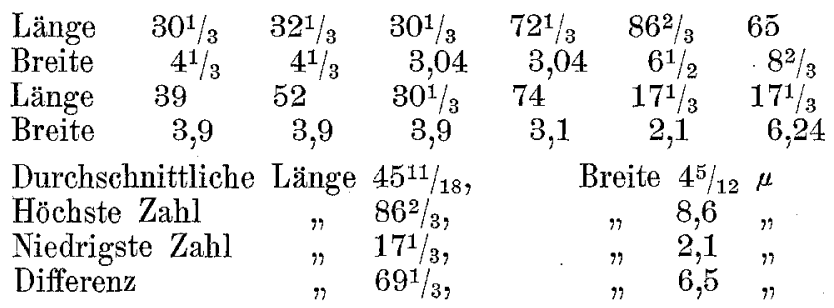

Es ist nun die Zahl der untersuchten Organe zu gering, um ein abschliessendes Urtheil über das Verhalten der Uterusmuskulatur bei entzündlichen Vorgängen zu erhalten, und es ist auch einigermaassen störend, dass in dem einen Falle ein Schleimpolyp im Fundus sass, im anderen zu dem Schleimpolypen noch ein kleines subseröses Myom kam. Doch glaube ich nicht, und die bisherigen Erfahrungen bestätigen dies, dass ein haselnussgrosses ganz subseröses Fibromyom von Einfluss auf die Uteruswand ist. Endometritisch veränderte Schleimhaut und ein Schleimpolyp sind aber nahe verwandte Dinge. Immerhin müsste zugegeben werden, dass auch der kleinste Fremdkörper im Uteruscavum den auslösenden Reiz für das Inkraftreten der immanenten Anlage des Organs werden könnte, da ja auch in den ersten Monaten der Schwangerschaft das Verhältniss zwischen Fruchtgewicht und Uterusgewicht ein anderes ist als später. Die Wirkung von im Uteruscavum befindlichen Fremdkörpern wird später eingehend besprochen werden. Ich würde, wenn Vergrösserungen der Muskelzellen in diesem Uterus mit Schleimpolypen nachgewiesen wären, keine Schlüsse ziehen dürfen, da dies aber nicht der Fall ist, können die Schleimpolypen unberücksichtigt bleiben.

Es ist nach den vorstehenden Untersuchungen wahrscheinlich, dass die Grösse der Muskelzellen je nach dem Stadium der entzündlichen Veränderung in der Uteruswand wechselt. Auch kommt wohl das Alter und der allgemeine Körperzustand der erkrankten Frau in Betracht. Leider fehlt ein Fall im Anfangsstadium. Uterus 3 stellt den Uebergang vom akuten Stadium zum chronischen dar.

Die Trägerin dieses Uterus war vor der Exstirpation ein Jahr lang krank. Uterus 1 enthält schon kein zellreiches Bindegewebe mehr, doch ist die eigentliche narbige Verhärtung desselben noch nicht eingetreten. Im Uterus 2 hat das sich retrahirende Binde- 
gewebe eine Umschnürung der Muskelzellen und ihrer Gefässe bewirkt, sodass Atrophie der muskulären Elemente, d. h. Verkleinerung der einzelnen, eingetreten ist. Mitgewirkt wird aber auch wohl die Krebskachexie der Patientin zur Verkleinerung der Muskelzellen haben. Vergleicht man die erhaltenen Zahlen mit den im normalen Uterus gefundenen, so tritt die Schwierigkeit hervor, dass die Grösse dieser auch nicht als völlig festgestellt betrachtet werden darf. Es kommen auch hier offenbar Schwankungen sowohl in Bezug auf das Individuum, wie auf die einzelne Muskelzelle vor.

Sänger giebt in der Festschrift für E. L. Wagner für den ruhenden, normalen Uterus zweier Frauen, die geboren hatten, folgende Zahlen:

1. Uterus.

Länge: Breite:

Höchste Zahl . . .

$49,4 \mu$

$6,5 \mu$

Niedrigste Zahl . . .

$15,6 \mu$

$2,6 \mu$

Durehschnitt aus je

10 Zahlen...

$29,9 \mu$

$4,4 \mu$

2. Uterus.

Höchste Zahl . . .

Niedrigste Zahl . . .

Durchschnitt aus je

$46,8 \mu$

$6,5 \mu$

$20,8 \mu$

$3,9 \mu$

10 Zahlen....

$38,3 \mu$

$5,7 \mu$

Kölliker giebt an, dass die Länge der Muskelzellen im ruhenden Uterus $44-68 \mu$, ihre Breite $4,5 \mu$ beirage, das Mittel aus 44 und 68 beträgt $51 \mu$, doch ist diese Zahl wohl kaum so zu verwerthen.

Ich fand im jungfräulichen Uterus folgende Zahlen:

$\begin{array}{lll} & \text { Länge: } & \text { Breite: } \\ \text { Höchste Zahl . . . } & 74,3 \mu & 4,3 \mu \\ \text { Niedrigste Zahl . . . } & 21,3 & 2,3 \\ \text { Durchschnitt aus je } & & \end{array}$

12 Zahlen ...

38,41

3,62

Vergleicht man diese Zahlen mit den bei den erkrankten Organen erhaltenen, so stellt sich heraus, dass bei Uterus 2 eine wesentliche Verkleinerung stattgefunden hat, doch muss man hier die vorhandene Krebskachexie in Rechnung ziehen. Uterus 1 scheint sich in der Grösse der Muskelzellen nicht besonders vom normalen Uterus zu unterscheiden. Uterus 3 zeigt eine geringe Verlängerung der Muskelzellen, sie haben durchschnittlich 


\begin{tabular}{|c|c|c|c|c|}
\hline \multirow{3}{*}{ gegen } & \multicolumn{2}{|c|}{$45^{11 / 18} \mu$ Länge, } & \multicolumn{2}{|c|}{$4^{5} / 13 \mu$ Breite, } \\
\hline & 29,9 & $n$ & 4,4 & $n$ \\
\hline & 38,3 & $n$ & 5,7 & " \\
\hline & 38,41 & 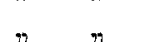 & 3,62 & ק \\
\hline
\end{tabular}

nach Sänger's und nach meinen Messungen.

Zu berüeksichtigen ist ferner, dass im Uterus 3 ein grosser Unterschied zwischen den einzelnen Muskelzellen hervortritt. Es wurden in ihm sowohl sehr kleine wie sehr grosse Muskelzellen gemessen, die Differenz zwischen der längsten und kürzsten betrug $69,3 \mu$, zwischen der breitesten und schmalsten $6,5 \mu$; combinirt man diese Zahlen mit den an Schnittpräparaten gefundenen Verhältnissen, so wird es wahrscheinlich, dass hier folgendes vorliegt: Neben der Bildung neuen Bindegewebes geht eine Neubildung von Muskelzellen einher; die Muskelzellen sind zum Theil, und zwar da, wo sie noch nicht von Bindegewebe umschnürt werden, hypertrophisch, d. h. im einzelnen vergrössert, wo sie von später sich bildendem festen Bindegewebe umgeben worden sind, sind sie normal oder verkleinert.

Im Uterus 2 scheint mir bei Vergleich mit einem jungfräulichen Uterus eine Verminderung der Muskelsubstanz zu Gunsten des Bindegewebes, im Uterus 3 eine theilweise Vermehrung und Vergrösserung der Muskelzellen, im Uterus 1 insofern ein eigenthümliches Verhältniss vorzuliegen, als bei normal grossen Muskelzellen einzelne Bündel durch Bindegewebe auseinander gedrängt, andere normal geblieben sind. Darnach würde sich die Beurtheilung der Zustände richten, wenn man, wie oben ausgeführt, Uterus 2 als Beispiel einer abgelaufenen chronischen Metritis, Uterus 1 als Beispiel einer chronischen Metritis und Uterus 3 als Beispiel einer subacuten chronischen Metritis auffasst. Eine Vermehrung der Muskulatur im Verhältniss zum Bindegewebe liegt nirgends vor, eine absolute Vermehrung nur im Uterus 3.

Ich gehe jetzt zur Mittheilung meiner Unterstichungen über das Verhalten der Uterusmuskulatur bei krankhaften Geschwülsten destructiven Charakters, also Carcinomen, Sarcomen, Deciduomen über.

4. Uterus von einer 47 jährigen Frau.

Dieses Organ wurde wegen eines Cervixcarcinoms exstirpirt. Die Cervix ist durch Zerfall von Tumormassen ganz verschwunden, die Schleimhaut weit hinanf krebsig verändert. Der Rest des Uterus besitzt $11 \mathrm{~cm}$ Länge, seine Wände sind $2 \mathrm{~cm}$ dick, grauroth auf dem Durchschnitt und fühlen sich fest an. Eine Schichtung ist nicht in ihnen zu 
erkennen, ihr Gewebe erscheint als ein gleichmässiges Filzwerk. Die mikroskopische Untersuchung ergiebt, dass an der gewöhnlich zur Untersuchung benutzten Stelle krebsige Veränderungen schon in die Muskulatur eindringen, dass spärliche, zum Theil schlecht gefärbte Muskelbündel von straffem Bindegewebe überwuchert wurden. Hier und da sieht man Rundzelleninfiltration. Auch hier ist die Messung an Querschnitten sehr sehwierig, doch lässt sich feststellen, dass keine Vergrösserung sondern eher eine Verkleinerung der Muskelzellquerschnitte besteht. Die höchste gemessene Zahl beträgt $4 \mu$, die kleinste $2 \mu$. An Zerzupfungspräparaten wurden an einer Stelle folgende Zahlen ermittelt:

\begin{tabular}{|c|c|c|c|c|c|c|}
\hline $\begin{array}{l}\text { Länge: } \\
\text { Breite: } \\
\text { Länge: } \\
\text { Breite: }\end{array}$ & $\begin{array}{c}52 \\
4,3 \\
212 / 3 \\
2,3\end{array}$ & $\begin{array}{c}52, \\
3,5, \\
21^{2} / 3 \\
3,4,\end{array}$ & $\begin{array}{c}51^{2} / 3 \\
3,0 \\
21^{2} / 3 \\
3,1\end{array}$ & $\begin{array}{c}43, \\
4,3, \\
26, \\
3,9,\end{array}$ & $\begin{array}{r}65, \\
4,3, \\
21^{2} / 3 \\
2,3\end{array}$ & $\begin{array}{c}65, \\
6,5, \\
26,\end{array}$ \\
\hline \multirow{2}{*}{\multicolumn{2}{|c|}{$\begin{array}{l}\text { Durchschnittliche } \\
\text { Höchste Zahl }\end{array}$}} & te Länge & $39^{7}$ & a, & Breite: &, 7 \\
\hline & & . & & $"$ & $"$ & 5 \\
\hline \multirow{2}{*}{\multicolumn{2}{|c|}{$\begin{array}{l}\text { Niedrigste Zahl } \\
\text { Differenz }\end{array}$}} & " & $21^{2 / 3}$ & 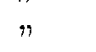 & $"$ & 3 \\
\hline & & $n$ & & n & $n$ & \\
\hline
\end{tabular}

An anderen Präparaten, welche durch Zerzupfung höher gelegener Partien hergestellt waren, fanden sich neben kleineren auch Muskelzellen von 90-108 $\mu$ Länge und 6-8 $\mu$ Breite, das Durchschnittsmaass betrug hier $50-60 \mu$ in der Länge und $5 \mu$ in der Breite. Schnittpräparate von dieser Stelle ergaben, dass die Wand hier zum grossen Theil aus Muskelbündeln zusammengesetzt war, innerhalb derer aber die Bindegewebsscheiden verbreitert waren, sodass an manchen Stellen die Muskelzellen in festes Bindegewebe eingelagert erschienen.

Durchmesser an Querschnitten, welche bis zu $8 \mu$ betrugen, wurden besonders da gemessen, wo die Verbreiterung der Bindegewebsscheiden weniger hervortrat. Frische entzündliche Veränderungen sind hier nicht zu sehen.

\section{Uterus von einer 40jährigen Frau.}

Dieser Uterus wurde wegen eines papillären Portiokrebses exstirpirt. Die Veränderung durch den Krebs reicht auf der Hinterfläche des Organs bis $3 \mathrm{~cm}$ unterhalb der Tubenansätze, mehr nach vorn und seitlich ist sie auf die Portio beschränkt. Der Uterus hat eine Länge von $10 \mathrm{~cm}$ und eine Wanddicke von $3 \mathrm{~cm}$.

Die Wände sehen in der Nähe der Schleimhaut gleichmässig verfilat aus. Nahe der Serosa ist bei einem grossen Theile des Organs auf $1 / 2 \mathrm{~cm}$ Breite deutliche. Schichtung zu sehen.

Die mikroskopische Untersuchung ergiebt an verschiedenen Stellen. verschiedene Resultate. An einem Theil der Schnitte sieht man reichliche engliegende Muskelbündel, die sich durch grossen Kernreichthum und verschiedene Länge ihrer Kerne auszeichnen, in der Umgebung derselben und in ihnen selbst kernreiches hyperplasirendes Bindegewebe. Auch in der Nähe der Gefässe sieht man kernreiches Bindegewebe. Rundzelleninfiltrationen sind mit Sicherheit nirgends zu erkennen. An anderen Stellen ist das Bindegewebe sowohl in der Nähe der Gefässe, wie in der Ūmgebung der Muskelbündel und in diesen selbst ärmer an Zellen und macht mehr einen homogenen Eindruck. An einer dritten Stelle ist die Wand aufgelockert. Zwischen Muskelbündeln, in denen 
die durchschnittliche Dicke der Muskelzellen $5-6 \mu$ beträgt, sieht man lockeres, sehr zellreiches Bindegewebe, die Auflockerung des Gewebes und die Vergrösserung der Muskelzelldurchmesser tritt besonders in der mittleren Schicht hervor. Die Messungen an Zerzupfungspräparaten ergeben demgemäss sehr verschiedene Resultate. Ich führe deshalb hier mur Messungen der 6 grössten und 6 kleinsten Muskelzellen an.

\begin{tabular}{|c|c|c|c|c|}
\hline Länge: $871 / 3$, & $108^{1 / 3}$ & $108^{1 / 3}$, & $108^{1} / 3$ & $130, \quad 99^{2} / 3 \mu$ \\
\hline Breite: 8,6, & 4,3 & 6,5 & 4,0 & $5,0,4,3$ \\
\hline Läuge: $171 / 2$, & $211 / 3$ & $171 / 3$ & $301 / 3$ & $211 / 3, \quad 26$ \\
\hline Breite: 8,2 , & 4,0 & 2,6 & 3,2 & $3,9,2,34$ \\
\hline $\begin{array}{l}\text { Höchste Zah } \\
\text { Niedrioste }\end{array}$ & & & $30 \quad \mu$ & Breite 8,6 \\
\hline Differenz & & & $122 / 3$ & 6,0 \\
\hline
\end{tabular}

6. Uterus von einer 44 jährigen Frau.

Auch dieser Uterus wurde wegen Cervixcarcinoms entfernt. Der Rest des Organs - die Portio war durch Zerfall der Neubildung und ärztliche Eingriffe ganz verschwunden - misst $9 \mathrm{~cm}$ in der Länge und seine Wanddicke beträgt $2-2^{1 / 2} \mathrm{~cm}$. Die Wand sieht grauröthlich aus und fühlt sich fest an. Eine Schichtung der Muskelsubstanz ist nicht zu bemerken. An der Schleimhaut reicht die Neubildung, welche sich als Adenocarcinom bei der Untersuchung erwies, bis dicht unter die Tubenmündungen, doch hat sie hier nur oberfiächliche Zerstörungen gemacht.

An Schnittpräparaten ist die Wand, mit Ausnahme der Umgebung grösserer Gefässe, überwiegend aus Muskulatur zusammengesetzt, welche sich aber dadurch von der normalen unterscheidet, dass innerhalb der Muskelbündelchen und um dieselben herum die Bindegewebszüge verbreitert und homogen erscheinen. Auch in der Nähe der grösseren Gefässe sieht man verbreitertes Bindegewebe mit kaum erkennbarer Faserung. Hier und da ist noch geringfügige, frische Bindegewebswucherung $\mathrm{zu}$ erkennen. Schnitte von verschiedenen Stellen zeigen hier eine grössere Gleichmässigkeit. An manchen ist aber auch in diesem Falle noch frische Bindegewebswucherung innerhalb der Muskelbündel und um diese herum zu sehen. Frische entzïndliche Veränderungen sind nicht vorhanden, Rundzellenhaufen sind nicht mit Sicherheit nachweisbar. An einigen Stellen ist auch eine Vergrösserung der Muskelzellen geringeren Grades eingetreten. Dort ist weniger Bindegewebe als normal. Die Querschnitte reichen an diesen Stellen in seltenen Fällen bis an 8 heran. An Zerzupfungspräparaten, welche auch nicht ganz gleichmässige Resultate ergeben, erhielt ich folgende Zahlen.

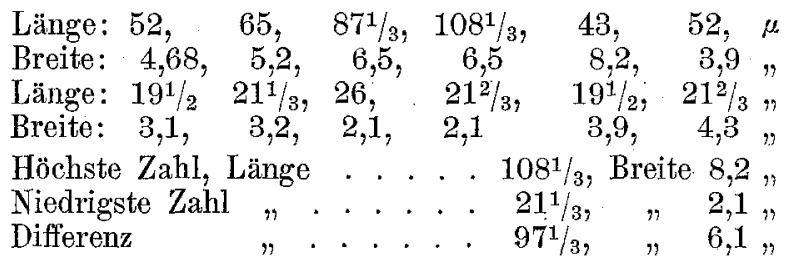

7. Uterus von einer 38 jährigen Frau.

Dies Organ wurde wegen malignen Cervix-Adenoms exstirpirt. Die Cervix an demselben war fast ganz versehwunden. Der Rest des Organs 
maass $10 \mathrm{~cm}$ in der Länge, seine Wände waren $\dot{2} \mathrm{~cm}$ dick, grauroth, fest und zeigten keine Sehichtung.

Die mikroskopische Untersuchung der theils verdickten, theils mit ïbelriechenden Zerfallsmassen bedeckten Schleimhaut des Fundus ergab, dass Drüsenwucherungen mit die Umgebung infiltrirendem Charakter anch an der gewöhnlich zur Untersuchung benutzten Stelle noch vorhanden waren. An Schnittpräparaten sieht man, dass die Wand überwiegend aus Muskulatur besteht, mit Ausnabme der Stellen, wo grössere Gefässe verlaufen, ist das Bindegewebe vermehrt. Innerhalb der Muskelbündelchen, welche sehr zellreich sind und verschiedene lange Kerne enthalten, sieht man bedeutende Zellvermehrung im Bindegewebe, ohne dass infiltrirende Rundzellen deutlich als solche zu erkennen wären. Näher der Serosa ist dieses Bindegewebe weniger zellreich und etwas breiter als normal. An mehreren Stellen sind die Durchmesser der Muskelzellquerschnitte vergrössert, sie halten $6-8 \mu$ in einzelnen Fällen. Zerzupfungspräparate ergeben mit ziemlicher Regelmässigkeit folgende Maasse.

\begin{tabular}{|c|c|c|c|c|c|c|}
\hline $\begin{array}{l}\text { inge: } \\
\text { eite: } \\
\text { inge: } \\
\text { eite: }\end{array}$ & $\begin{array}{c}52 \\
4,0 \\
21^{2} / 3 \\
6,96\end{array}$ & $\begin{array}{c}65, \\
4,3 \\
26, \\
3,9\end{array}$ & $\begin{array}{c}43, \\
4,0, \\
21, \\
5,5\end{array}$ & $\begin{array}{c}86^{1} / 2, \\
6,5, \\
17^{1} / 3, \\
3,9,\end{array}$ & $\begin{array}{c}47^{2} / 3, \\
6,8, \\
21^{2} / 3 \\
6,2\end{array}$ & $\begin{array}{rr}78 \mu \\
4,3 \\
27^{2} / 3 \\
3,9 \\
3,\end{array}$ \\
\hline & & & & $\begin{array}{l}42^{1} / 3, \\
86^{1} / 3, \\
17^{1} / 3, \\
69,\end{array}$ & Breite & $\begin{array}{r}\text { e } 5,5 \\
6,9 \\
3,9 \\
3,0\end{array}$ \\
\hline
\end{tabular}

Die vorliegenden Untersuchungen ergaben, dass im Uterus bei Carcinomen sehr verschiedene. Veränderungen stattfinden können. Als allgemeingültig kann aber der Satz aufgestellt werden, dass in erster Linie eine Hyperplasie der sämmtlichen Gewebselemente stattfindet, ohne dass überall frische entzündliche Veränderungen nachzuweisen sind. Es wuchert Bindegewebe und Muskulatur gleichzeitig, das Ergebniss dieser Wucherung ist später ein Gewebe, welches dem normalen häufig nicht unähnlich ist, nur ist das Bindegewebe homogener, weniger faserig als im normalen Uterus. Auch ist es meistens sowohl innerhalb der Muskelbündelchen wie um die Gefässe herum verbreitert. Dort wo man frische Wucherungen fand, findet man, wie im Falle 3, auch sehr verschieden grosse Muskelzellen, unter denen einige immer bedeutende Dimensionen erreichen. Endlich kommt auch neben den hyperplastischen Vorgängen und sich mit diesen an derselben Stelle vereinigend eine Veränderung des Uterus vor, welche sich durch Auflockerung und Schichtung des "Gewebes den Veränderungen nähert, welche der Uterus bei Schwangersehaft eingeht, wenn auch die Grössenmaasse hinter denen bei Gravidität gefundenen zurückbleiben. Es lässt sich dies vielleicht dadurch erklären, dass das 
Cervixcarcinom ähnlich wie ein Pyloruskrebs dureh Stenosirung des Ostiums das Abfliessen des bei Krebs ja reichlicher abgeschiedenen Blutes und Schleimes verhindert oder erschwert. Der Uterus würde dann Contractionen machen müssen, um sich seines Inhaltes zu entledigen und dadurch eine Arbeitshypertrophie eingehen. Analog den übrigen Organen mit glatter Muskulatur würde sich dann der Uterus durch Vergrösserung der einzelnen Muskelzellen den erhöhten Anforderungen anpassen. So erklären sich die Ergebnisse der Zerzupfungspräparate. Um definitiv zu entscheiden, was von den besprochenen Veränderungen auf die Einwirkung des Tumors selbst und was auf die durch den Tumor secundär veranlasste Secretretention zu schieben ist, müsste man klinisch genau in dieser Hinsicht beobachtete Fälle haben. Ich glaube aber; dass wahrscheinlich die oben ausgesprochenen. Vermuthungen sich als rịchtig erweisen werden.

Das Verhältniss von Bindegewebe zu Muskulatur ist an verschiedenen Stellen und in verschiedenen Organen ein sehr verschiedenes, so dass es unmöglich ist, zu sagen, welches von beiden Gewebselementen bei den hyperplastischen Vorgängen. in vorwiegender Weise gebildet wird. Bei jüngeren Franen ist im Allgemeinen die Entwicklung der Muskelsubstanz bedeutender, wenn man das Verhältniss im ganzen Organ in Betracht zieht. Wenn man einzelne Stellen ins Auge fasst, so ist, wo frische Wucherung besteht, die Muskulatur meistens vorwiegend, während in späteren Stadien dieselbe innerhalb des homogen werdenden, sich retrahirenden Bindegewebes an Masse geringer wird.

Bei den bis jetzt untersuchten Fällen handelt es sich um entzündlichen Reizzustand, wenn ich die durch ein ulcerirendes Carcinom für die Umgebung gesetzten Veränderungen mit unter diesen Begriff fassen darf, bei welchem angenommen werden dart, wenn man von den Folgezuständen der Stenosirung des Cervicalkanals absehen will, dass theils durch die Entzündung selbst, theils durch die von ihr hervorgerufene. Congestionshyperämie auch in der näheren Nachbarschaft, die Vergrösserung und Vermehrung. der Gewebselemente verursacht wird.

Ich füge zwei Fälle an, bei denen am Uterus ein Tumor war, der ebenso wie das Carcinom maligne Natur hatte, aber durch seinen Sitz und seine Gestalt im Stande war, andere als entzündliche Reize auszuüben, nämlich den auslösenden Reiz zur Bethätigung der immanenten Anlage zưr Schwangerschaftsvergrösserung. 
Dieselbe d. h. die Anlage tritt in Kraft, wenn ein befruchtetes Ei sich im Uterus festgesetzt hat, dann vergrössert sich der Uterus, durch welche mechanische oder nervöse Reize ist nicht aufgeklärt.

Ein ähnlicher Reiz muss ausgeübt werden, wenn im Uterus sich ein in die Uterushöhle, ähnlich wie die Frucht hineinwachsender Tumor befindet, oder ein Fremdkörper, wie Blut u. s. w., der sich langsam vergrössert. Solche Verhältnisse bieten die beiden folgenden Fälle.

8. Uterus. Sammlungspräparat, eingelegt 1882 in Alkohol. Alter der Patientin 58 Jahre.

Der vorliegende Uterus ist im Orth'schen Lehrbuch Seite 486 abgebildet.

Von der Hinterwand des Fundus ragt ein knabenfaustgrosses Sarkom bis zum inneren etwas dilatirten Muttermund herab; der Uterus selbst misst $13 \mathrm{~cm}$ in der Länge, seine Wand ist $1 \frac{1}{2}-2 \mathrm{~cm}$ dick, diese zeigt eine zwar nicht sehr ausgeprägte, aber deutlich erkennbare Schichtung und fühlt sich ziemlich hart an, was wohl durch den langen Aufenthalt in Alkohol erklärt wird. Leider liess sich an dem alten Präparat keine gute Kernfärbung mehr erzielen, doch ist gut erkennbar, dass die Wand ihre vermehrte Dicke der Vermehrung der Muskelsubstanz verdankt. Besonders sind die der Serosa nahe gelegenen Schichten fast rein muskulär, hier finden sich auch deutlich hypertrophische Muskelzellen, welche in verschiedenen Bündelchen bei weitem die. Mehrzahl bilden. Es wurden in grosser Anzahl Muskelzellen von Durchmessern zwischen 8-13 $\mu$, in geringer Anzahl solche von $17 \mu$ gemessen. Je näher man der Schleimhaut kommt, desto kleiner werden die Durchmesser und sehliesslich kommen keine Durchmesser über $3-4 \mu$ mehr vor. Zerzupfungspräparate gelangen, wohl wegen des Alters des Präparates, leider nicht. Es kann aber auch so festgestellt werden, dass es sich um eine muskuläre Hypertrophie mit gewaltiger Dickenzunahme der Muskelquerschnitte handelt.

9. Uterus von einer 33jährigen Frau.

Die Patientin hatte im October 1894 einen Abort durchzumachen, litt seither an starken Blutungen, wegen deren sie im Anfang des Jahres 1895 in der Klinik behandelt, vorläufig entlassen und später wieder aufgenommen wurde.

Am 29. 5. ist ihr Uterus exstirpirt. Derselbe enthielt im Fundus einen Hühnerei grossen Tumor, welcher sich als Deciduom erwies, das Organ hat eine Länge von $13 \mathrm{~cm}$, seine Wand ist $2 \mathrm{~cm}$ dick, dieselbe ist grauroth and zeigt auf dem Durchschnitt eine feine Streifung. Die Consistenz des Organs ist eine mittelweiche. An Schnittpräparaten erkennt man, dass die Wand des Organs vorwiegend durch Muskulatur gebildet wird, dieselbe ist zu zahlreichen längsgetroffenen, parallel verlaufenden Muskelbündeln angeordnet, zwischen welchen man aber auch in grosser Anzahl schräg und quer getroffene Muskelbündel sieht. Die Muskelbündel sind durch geringere Mengen lockeren sehr zellreichen 
Bindegewebes mit einander verbunden und in diesem, besonders um die vielen Gefässe herum, liegen grössere Mengen von Rundzellen. Die Musculatur ist hypertrophisch.

An Querschnitten werden Durchmesser bis zu $11 \mu$ gemessen. Im Allgemeinen scheint die Dicke der Muskelzellen $7-8 \mu$ zu betragen.

An Zerzupfungspräparaten erhielt ich folgende Zahlen.

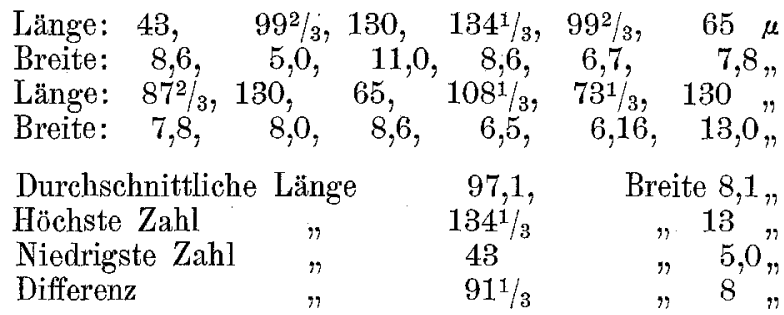

Von den vorliegenden zwei Fällen von polypöser maligner Tumorbildung bietet der erste ein gutes Beispiel einer muskulären wahren Hypertrophie in Folge eines abnormen Inhalts der Uterushöhle, denn trotz des zerstörenden Characters des Sarcoms haben sich die Muskelzellen auf den Reiz hin, welchen das Sarcom ausübte, vergrössert.

Der zweite kann ebenfalls als Beispiel einer muskulären Hypertrophie durch fremdartigen Inhalt in der Uterushöhle veranlasst betrachtet werden. Es gesellen sich hier noch Reize entzündlicher Art hinzu, welche durch die Malignität des Tumors bedingt werden. Auf dieselben lassen sich die hyperplasirenden Vorgänge im Bindegewebe zurückführen. Doch ist auch die Erklärung zulässig, dass die Involution durch Zurückbleiben von Placentarresten in diesem Falle verhindert worden sei, oder dass die Wucherung der Decidua an sich, ohne dass der Fremdkörperreiz hinzukäme, einen nahezu physiologischen Reiz auf die Uteruswand ausgeübt habe.

Man könnte die vorliegende Hypertrophie also auch entweder als abnorme Erhaltung eines physiologischen Zustandes oder als abnorme Entstehung des physiologischen Zustandes durch Wucherung von Eihäuten auffassen.

Tch komme jetzt zur Untersuchung der Uteruswand bei interstitiellen und submucösen Fibro-Myomen. Besonders erscheinen von vornherein die submucösen Myome geeignet, durch ihre eigenthümliche Lage zum Uterus Einflüsse auszuüben, welche den in der Schwangerschaft das Organ treffenden Erregungen ähnlich sind. Ebenso ist es mit den interstitiellen Myomen, sobald sie einige Grösse erlangt haben und dann eine dünne Schicht Mus- 
kulatur nebst Schleimhaut vor sich herschiebend, den submucösen Myomen bis auf die dünne sie überziehende Schicht Muskulatur durchgehends gleiche Verhältnisse setzend in die Fundushöhle hineinragen. Ganz andere Verhältnisse bieten die interstitiellen multiplen Myome dar, welche sich in grossen Mengen im gleichen Organ entwickeln und so viel Raum für sich in Anspruch nehmen, dass die Uteruswand oft nur aus Myomen, welche durch kurze Verbindungsbrücken von Uterussubstanz verbunden sind, besteht. Derartige Fälle wurden drei untersucht.

\section{Uterus. Stammt von einer 47 jährigen Frau.}

Es handelt sich hier um eine Combination von Careinom und multiplen Myomen. Ein Cervixcarcinom hat die ganze Portio umgriffen. Der Rest des Organs ist $12 \mathrm{~cm}$ lang, seine Wand da, wo sie nicht durch Fibro-Myome verändert ist, $2^{1 / 2} \mathrm{~cm}$ Dick. In der Wand des Fundus befinden sich zwei hühnereigrosse Fibro-Myome, ein mehr nach der Serosa zu sich entwickelndes und ein in die Fundushöhle hineinragendes; 4. kleinere Tumoren theilen sich in die übrigen Gegenden der Wand. An gefärbten Schnitten sieht man, dass die Entwickelung des Bindegewebes in der Wand eine sehr bedeutende gegenüber der der Muskulatur ist. Um die Gefässe herum lagern sich breite bindegewebige Massen, während die schmalen Muskelbündelchen von zarteren Bindegewebssträngen umschlossen sind. Das Bindegewebe ist ein faseriges, zellarmes. An einigen Stellen sieht man vereinzelt Rundzellen; Mastzellen nirgends. Ton dem die Muskelbündel umschliessenden Bindegewebe aus ziehen sich reichliche feinere Bindegewebsfasern in die Muskelbündel hinein und trennen die Muskelzellen, so dass sie an manchen Stellen nur zu zweien und dreien zusammenliegen, manchmal ganz isolirt sind. An Muskelzellquerschnitten wurden durchschnittlich $4 \%$ als Durchmesser gemessen; vereinzelt kamen solche bis zu $6{ }^{1} / 2 \mu$ vor. Die Kerne stehen mittelweit, sind lang und schmal und haben ein kaum sichtbares Chromatingerüst. An Zerzupfungspräparaten erhielt ich folgende Zahlen.

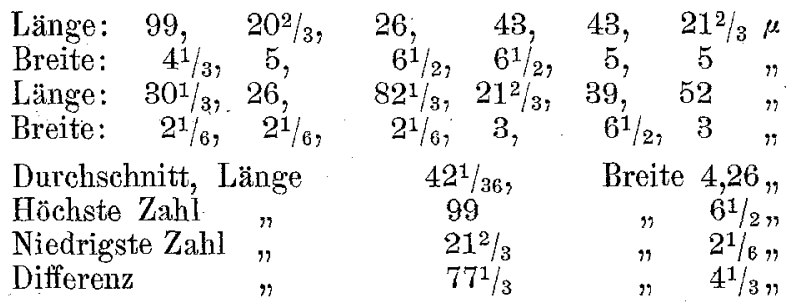

11. Uterus von einer 40 jährigen Frau.

Das Organ ist $14 \mathrm{~cm}$ lang und $7 \mathrm{~cm}$ breit. Seine Wand ist so von 7 ungefähr hühnereigrossen und verschiedenen kleineren Myomen durchsetzt, dass kaum eine Stelle zwischen den Tumoren übrig bleibt, um ein Stück zur Untersuchung zu entnehmen. Wo die Wand erhalten ist, ist sie gefässreich, weich und von graurother Farbe.

Unter dem Mikroskop sieht man an Schnittpräparaten nichts von der gewöhnlichen Abgrenzung der Muskelbündel durch Bindegewebe. 
Die Muskelzellen selbst erscheinen regellos durcheinander geflochten, hier und da sieht man zwischen ihnen zahlreiches Bindegewebe. Rundzellen und Mastzellen finden sich ebenfalls yor.

Messungen an Muskelquerschnitten geben kein sicheres Resultat. An Zerzupfungspräparaten wurden folgende Dimensionen gemessen.

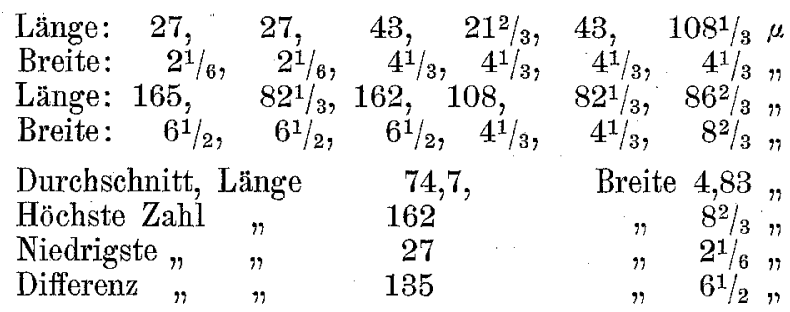

12. Uterus von einer 44 jährigen Frau.

Dieser Uterus bietet schon makroskopisch eigenthümliche Verhältnisse. Er hat Mannskopfgrösse, höckrige Oberfläche und seine Wände enthalten 11 Fibro-Myome bis zu Hühnereigrösse und darüber und viele kleine, welche sämmtlich nach allen Seiten von Muskulatur umgeben sind. Die Muskulatur ist, wo für sie zwischen den Tumoren ein Raum ausgespart ist, verdickt, sie misst $2-2^{1 / 2} \mathrm{~cm}$, zeigt eine deutliche Schichtung, ist gefasssreich und fühlt sich weich an. An der dicksten Stelle der Wand wurde ein Stück zur mikroskopischen Untersuchung entnommen, welches folgende Verhältnisse zeigt:

Die Schichtung der Muskellagen ist deutlich. zu erkennen. Jede Schicht besteht aus einem Muskelbündel, welches zu beiden Seiten lockeres Bindegewebe hat. In diesem bemerkt man hier und dort bald kleinere bald grössere Haufen von Rundzellen. Im Bindegewebe wie in der Muskulatur sieht man grosse Mengen von Mastzellen, welche im Bindegewebe rundliche, innerhalb der Muskulatur längliche Form haben. Bei Färbung mit polychromem Methylenblau sind diese letzteren Kerne, welche sonst bei Pikrolithionkarminfärbung Aehnlichkeit mit Muskelzellkernen haben, durch die intensiv violette Färbung, welche sie ebenso wie die in der Zelle liegenden Körner bekommen, deutlich unterscheidbar. Die Kerne der Muskelzellen sind gross und zeigen deutliches Chromatingerüst. Messungen an Querschnitten ergaben, dass die Durchschnittsbreite der Muskelzellen $7-8 \mu$ beträgt. An Zerzupfungspräparaten wurden folgende Zahlen gefunden.

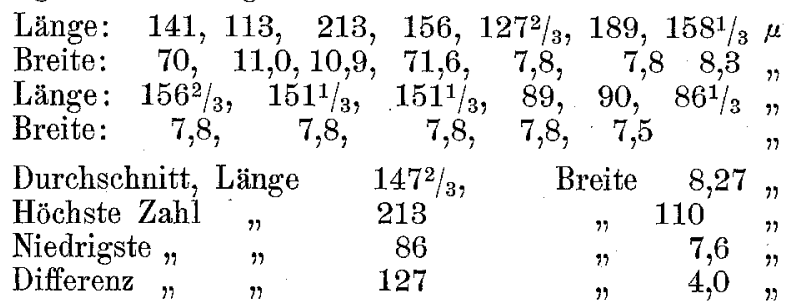

Die vorstehende Untersuchung der letzten drei Uteri mit multiplen interstitiellen Myomen ergab also, dass eine bestimmte Regel für das Verhalten der Uteruswand nicht aufzustellen ist. In dem Falle 10 war die Gesammtmenge der Uterussubstanz 
vermehrt, gegenüber der Gesammtmenge des normalen. Die Zahl und das Gesammtvolumen der Myome geringer als in den beiden folgenden Fällen. Eine Vermehrung der Wandsubstanz hatte auch im Fall 12 sich eingestellt. Im Fall 11 aber war es eine Verminderung. In allen Fällen war aber, dasjenige was zwischen den Myomen von Wand übrig geblieben, mächtig verdickt. Diese Verdickung kann man erstens als einen Zustand auffassen, wie ihn Labbé als "Hypertrophie Fibroso-myomateuse" beschreibt, wobei in zwei von diesen Fällen abweichend von den durch Labbé beschriebenen die Hypertrophie hauptsächlich eine myomatöse wäre. Damit würde auch das Vorwiegen des Muskelgewebes und die Vergrösserung der Muskelzellen gut übereinstimmen. Denn es kann wohl kein Zweifel sein, dass es sich, wenn ein Uterus von vielen Myomen durchsetzt ist, mehr um eine allgemeine Erkrankung des ganzen Organs, welche stellenweise stärker auftritt, als um eine mehrfach localisirte Hypertrophie, die man sonst wohl angenommen hat, handelt. Es bietet auch die Wand des Uterus 11 durch ihre filzartige Verflechtung von Muskelzellen untereinander und mit Bindegewebe ganz den Anblick eines Myoms dar. Natürlich könnte dieses fibro-myomatöse Hypertrophie auch eine mehr fibröse als muskuläre sein, was den Befund bei Uterus 10 ebenfalls erklärte. Anders liegt es aber bei Uterus 12, wo die regelmässig geschichtete Wand hypertrophische Muskelzellen zeigt. Hier muss man eine Arbeitshypertrophie durch Contractionen oder eine Erregung der immanenten Anlage zur Vergrösserung des Uterus wie in der Schwangerschaft durch die Tumoren zur Erklärung annehmen. Auch erscheint dies besonders in Fall 12 nicht von der Hand zu weisen, da die Tumoren nach ausserhalb überall von Muskulatur umgeben waren und nach innen zum Theil vorragten, wenn auch noch von einer dünnen Muskelschicht überzogen, also der Muskulatur sowohl einen Angriffspunkt boten, wie durch ihr Hineinragen in die Uterushöhle die immanente Anlage zur Bethätigung reizen konnten.

Es folgen jetzt die Resultate der Untersuchung der Uteruswand bei einfachen interstitiellen und submucösen Myomen und bei Hämatometra.

13. Uterus von einer 40 jährigen Frau.

Der Rest des durch supravaginale Amputation entfernten Organs misst vom Ansatz des Scheidengewölbes bis zum Fundus $10,5 \mathrm{~cm}$, seine Wand hat eine Dicke von $3-4^{1 / 4} \mathrm{~cm}$. In der Hinterwand etwas seit- 
lich von der Mittellinie befindet sich ein $6 \mathrm{~cm}$ im Durchmesser haltendes sehr hartes Fibro-Myom. Die Wände fühlen sich fest an und zeigen auf dem Durchschnitt eine gleichmässig verfilzte Structur. Bei mikroskopischer Untersuchung erweist es sich; dass diese Wände zum grossen Theil aus Bindegewebe bestehen. Schmale Muskelbündelchen von breiten Bindegewebszügen umgeben, werden selbst noch von verbreiterten Bindegewebsfasern durchzogen. Das Bindegewebe hat noch ziemlich viel Zellen, ist aber kein junges Bindegewebe mehr. In der Nähe der Gefässe sieht man hier und da entzündliche Infiltrationen und einzelne Mastzellen. Die Muskelzellen sind auf Querschnitten schmal, ihre Durchmesser betragen nicht über $4,5 \mu$, ihre Kerne sind klein und zeigen undeutliches Chromatingerïst. Zerzupfungspräparate geben folgendes Resultat.

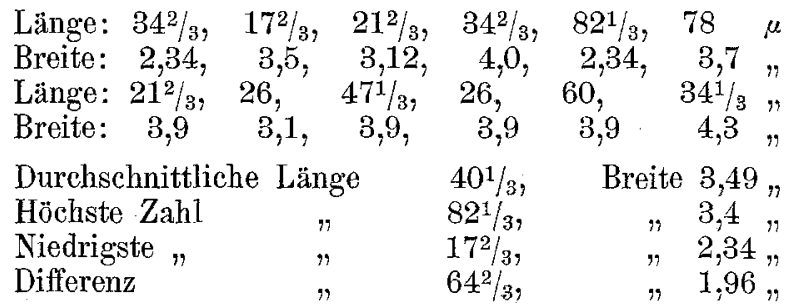

\section{Uterus von einer 44 jährigen Frau.}

Die Länge des amputirten Stücks des durch supravaginale Amputation entfernten Organs betrug $25 \mathrm{~cm}$. Die Wanddieke betrug an der dicksten Stelle $2{ }^{1 / 2} \mathrm{~cm}$. In der hinteren Wand vom Fundus entspringend befindet sich durch eine ziemlich schmale Muskelschicht von der Uterushöhle getrennt ein $13 \mathrm{~cm}$ im Durchmesser haltendes Fibro-Myom, etwas unterhalb desselben und mehr seitlich ein kleines submuköses Myom von $4 \mathrm{~cm}$ Durchmesser, welches aber nicht in die Fundushöhle einragt, sondern ungefähr in der Höhe des inneren Muttermundes sich entwickelnd das untere Uterinsegment ausdehnt. Die Wand sieht anf dem Durchschnitt gleichmässig verfilzt aus, ist grauröthlich und ziemlich hart. Mit dem Mikroskop erkennt man, dass dieselbe aus Muskulatur zu gleichen Theilen gemischt mit zellarmem Bindegewebe besteht, welches sowohl die einzelnen Bündel umschliesst, als auch sich innerhalb derselben verbreitert hat. Die Querschnitte der Muskelzellen sind hier ziemlich gross, sie messen durchschnittlich $6 \mu$, ganz vereinzelt sieht man auch solche, welche $10 \mu$ in die Breite messen. Frische Wucherungen, kleinzellige Infiltrationen und Mastzellen sind nicht vorhanden. Die Muskelzellkerne sind ziemlich schmal und zeigen ein erkennbares aber nicht sehr deutliches Chromatingerïst. Zerzupfungspräparate geben folgende Zahlen.

\begin{tabular}{|c|c|c|c|c|}
\hline $\begin{array}{lc}\text { Länge: } & 39, \\
\text { Breite: } & 7,5, \\
\text { Länge: } & 30^{1} / 3, \\
\text { Breite: } & 5,5,\end{array}$ & $\begin{array}{cc}42, & 55^{1} / 3, \\
10,0, & 8,9 \\
57^{2} / 3, & 86^{2} / 3 \\
6,2, & 4,3\end{array}$ & $\begin{array}{c}60, \\
4,3, \\
39 \\
4,3\end{array}$ & $\begin{array}{c}301 / 3 \\
7,8 \\
72^{1} / 3 \\
7,9\end{array}$ & $\begin{array}{c}42^{1 / 2} / 2 \\
5,45 \\
36^{1 / 3} \\
8,9\end{array}$ \\
\hline $\begin{array}{l}\text { Duchschnittliche } \\
\text { Höchste Zahl } \\
\text { Niedrig*ste " } \\
\text { Differenz }\end{array}$ & $\begin{array}{c}\text { e Länge } \\
n \\
n \\
n\end{array}$ & $\begin{array}{l}49^{1} / 4 \\
86^{2} / 3 \\
39^{1} / 3 \\
47^{1} / 3\end{array}$ & $\begin{array}{r}\text { Bre } \\
" \\
" \\
n\end{array}$ & $\begin{array}{r}6,6 \\
10,0 \\
4,3 \\
5,7\end{array}$ \\
\hline
\end{tabular}




\section{Uterus von einer 42 jährigen Frau.}

Dieses Organ, welches vom Ansatz des Scheidengewölbes bis zum Fundus $18 \mathrm{~cm}$ maass, trug in der hinteren Wand ein interstitielles Fibromyom von $8 \mathrm{~cm}$ Durchmesser, welches fast ganz in die Fundushöhle hineinragte und an seiner vorderen Fläche ausser von der Schleimhaut nur von einer ziemlich dünnen Schicht Muskulatur überdeckt wurde. Im übrigen waren die Wände des Uterus $2-2^{3 / 4} \mathrm{~cm}$ dick und ziemlich hart. Eine Schichtung war nicht zu erkennen, die Farbe weisslich- oder hell-grau-roth.

Am gefärbten Schnitt sieht man, dass nach der Schleimhaut zu schmälere, nächst der Serosa breiter werdende Muskelbündel von reichlich entwickeltem, zellarmen, straffen, faserigen Bindegewebe durchzogen werden. In der Nähe der Gefässe befinden sich zahlreiche Rundzellen im Gewebe, die grössten Durchmesser von Muskelzellen betrugen $8{ }^{2} / 3 \mu$, die Mehrzahl ging aber über $4-5 \mu$ nicht hinaus. Anch hier sind die Kerne schmal, sie haben undeutliches Chromatingerüst. An Zerzupfungspräparaten maass ich folgende Zahlen.

\begin{tabular}{|c|c|c|c|c|c|}
\hline $\begin{array}{lc}\text { ange: } & 51, \\
\text { reite: } & 4,3, \\
\text { reinge: } & 30, \\
\text { reite: } & 2,5,\end{array}$ & $\begin{array}{c}61, \\
6,5 \\
52, \\
6,5\end{array}$ & $\begin{array}{c}321 / 3, \\
4,3 \\
21 \% \\
4,3,\end{array}$ & $\begin{array}{c}65, \\
4,3, \\
61, \\
8,9,\end{array}$ & $\begin{array}{c}32, \\
4,3, \\
31, \\
6,5,\end{array}$ & $\begin{array}{c}56 \\
4,3 \\
30^{1} / 3 \\
3,0\end{array}$ \\
\hline & Län & & & $\begin{array}{c}\text { Breite } \\
n \\
n\end{array}$ & $\begin{array}{l}4,97 \\
8,9 \\
2,5 \\
6,4\end{array}$ \\
\hline
\end{tabular}

16. Uterus von einer 50 jährigen Frau.

In der Hinterwand des vom Ansatze des Scheidengewölbes bis zum Fundus $25 \mathrm{~cm}$ in der Länge messenden Organes befindet sich ein mannskopfgrosses Fibro-Hyom, welches die vor ihm liegende Schicht der Hinterwand vor sich her in die Fundushöhle hinein verschoben hat. Diese Schicht ist sehr dünn. Die Schleimhaut über ihr zeigt eine ungleichmässige, geschwulstartige Verdickung, von welcher die mikroskopische Untersuchung ergiebt, dass sie auf Endometritis proliferans zurückzuführen ist. Die Wand des Uterus ist $2-2^{1 / 2} \mathrm{~cm}$ dick, zeigt keine Trennung und Auflockerung in einzelne Schichten und füblt sich ziemlich fest an. Die Farbe ist weisslich-roth. Mit dem Mikroskop sieht man, dass sie aus Muskelbündeln, welche keine vergrösserten Muskelzellen enthalten und welche von vermehrtem Bindegewebe umgeben werden, besteht. Das Bindegewebe ist zellarm, faserig. Hier und da sieht man in den mit Pikrolithionkarmin gefärbten Schnitten bei schwacher Vergrösserung auffallend gelb gefärbte Stellen in der Muskulatur, welche, bei starker Vergrösserung, als umschriebene deutlich abgrenzbare hypertrophische Bezirke in der Muskulatur sich erweisen. Manchmal ist ein ganzes Bündel, manchmal nur ein kleiner Theil desselben von dieser Vergrösserung seiner Muskelzellen betroffen. Ausserdem sieht man bald quer bald schräg getroffene Hohlräume, welche in mit Müller'scher Flüssigkeit gefärbten Schnitten kleine Mengen hyaliner Massen ohne Kernfärbung, hier und da auëh rothe Blutkörperchen enthalten. Diese Hohlräume haben in ihren Wänden Verdickungen, welche aus Muskelbündeln bestehen, die wie die oben beschriebenen hypertrophisch sind. Manchmal geht um die dicken Wände, wenn der 
längliche Hohlraum und die hyertrophischen Muskelzellen in seiner Umgebung quer getroffen sind, aussen ein Gewebsring herum, dessen längsgetroffene Zellen Mediazellen ähnlich sind; manchmal scheint dieser Ring durchbrochen oder selbst in Wucherung übergegangen zu sein, sodass die vergrösserten Muskelwülste direct in die Umgebung einragen, an noch anderen Stellen sieht man folgendes Bild:

Die Hohlräume sind von graurother Intima ausgekleidet, auf diese folgt ein dicker der Media entsprechender Gewebsring mit längsgetroffenen Zellen, das Ganze wird im weiteren Umkreise von 1 bis 2 Reihen quergetroffener, also zum Hohlraum längsverlaufender hypertrophischer Muskelbündel umgeben. Die Muskelzellen in den hypertrophischen Bezirken messen 6-8-9 $\mu$, die in den normal gebliebenen $3-4 \mu$.

Die beschriebene Veränderung fand sich in Schnitten, welche von 4 verschiedenen Stellen des Organs angefertigt waren.

Muskelzellmessungen an zerzupften Wandstückchen gaben das erwartete Resultat, dass vergrösserte und normale oder verkleinerte Muskelzellen, zwischen denen es wenig mittelgrosse gab, gemessen wurde. Auf eine Berechnung der durchschnittlichen Grösse wurde deshalb verzichtet.

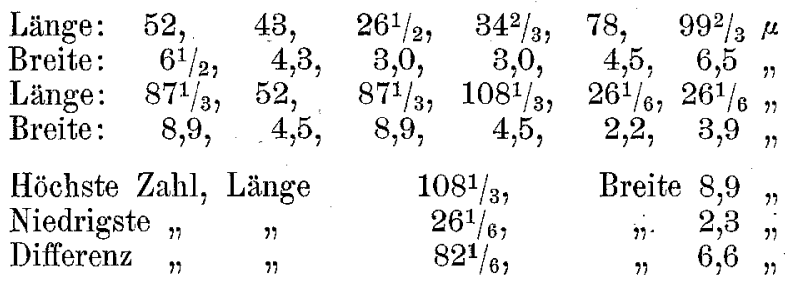

\section{Von einer 38 jährigen Frau.}

Dieser Uterus wurde durch supravaginale Amputation entfernt. Die Länge vom Fundus bis zum Ansatz des Scheidengewölbes betrug $18 \mathrm{~cm}$, die Wanddicke $2 \mathrm{~cm}$. Die Hinterwand enthielt ein $15 \mathrm{~cm}$ im Durchmesser haltendes Fibro-Myom, welches die Muskulatur der mittleren und seitlichen Partien des Uterus stark nach der Fundushöhle zu vorgedrängt hatte, aber allseitig von Muskulatur umgeben war. Die Wand fühlte sich ziemlich fest an, zeigte eine Schichtung nur etwas angedeutet, sie sieht weisslich-roth aus. Im Tumor ist ausgedehnte fettige Degeneration nachzuweisen. Die mikroskopische Untersuchung ergiebt, dass die Wände aus Muskulatur gleichmässig gemischt mit Bindegewebe bestehen. Die erstere ist zu Bündeln angeordnet, in welche das Bindegewebe in verbreiterten Zügen hineindringt, sodass bei schwacher Vergrösserung: wie muskulär aussehende Bündel sich mit starker Vergrösserung in Muskelzellen, von denen jede einzelne von verbreitertem Bindegewebe umgeben ist, auflösen lassen. Das Bindegewebe ist dicht, zellarm und von einer Auflockerung der Wand und Schichtung der Bestandtheile ist nichts zu sehen. Entzündliche Veränderungen fehlen. Messungen an Muskelquerschnitten sind in dem straffen Gewebe nicht leicht zu bewerkstelligen. Die Durchschnittsmaasse scheinen . $5-6 \mu \mathrm{zu}$ betragen. Hier und da misst man auch Muskelzellen von 8-9 $\mu$. Durchmesser. An Zerzupfungspräparaten erhielt ich folgende Werthe. 


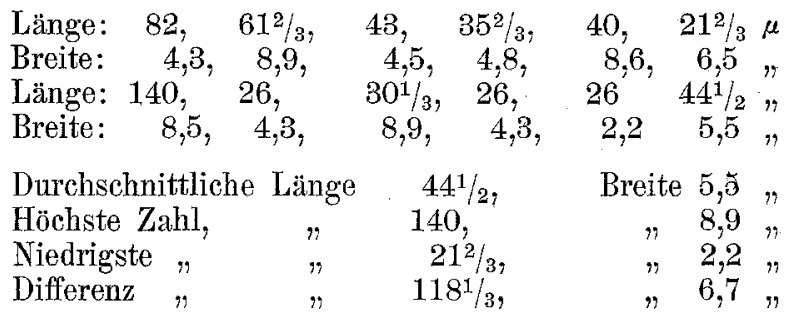

\section{Von einer 42 jährigen Frau.}

Dieses Organ ist $25 \mathrm{~cm}$ lang, vom Ansatz des Scheidengewölbes bis zum Fundus gemessen. Seine Wanddicke beträgt in der vorderen Wand bis $z \mathfrak{u} 3$, in der hinteren bis $\mathrm{zu} 4 \mathrm{~cm}$. In der hinteren Wand hat sich ein $12 \mathrm{~cm}$ im Durchmesser haltendes Fibro-Myom gebildet, welches den vordern Theil der Wand weit nach vorn und unten gedrängt hat. Die Schleimhaut ist endometritisch verändert, die vordere Wand ist ziemlich fest ohne deutliche Schichtung und Auflockerung und weisslichroth gefürbt; die hintere Wand ist ganz aufgelockert und zeigt sehr auffallende Schichtung in dicke Muskellagen, zwischen denen sich lockeres Bindegewebe und weite Gefässe befinden. Die Uterushöhle ist verhältnissmässig wenig erweitert; sie liegt gewissermaassen dem Tumor auf. In der hinteren Wand finden sich noch verschiedene erbsengrosse und noch kleinere Hyome. Auf Quersehnitten durch die hintere Wand sieht man, dass die Schichtung bei schwacher Vergrösserung noch deutlich zu erkennen ist. Längsgetroffene Muskelbündel, zwischen denen sich einige quergetroffene befinden, werden von lockerem, gefäss- und zellreichem Bindegewebe zusammengehalten. Bei etwas stärkerer Vergrösserung erkennt man leicht, dass eine allgemeine Vergrösserung der Muskelzellen vorliegt. Messungen an Querschnitten ergaben, dass die Durchschnittsgrösse der Muskelzellen $6-9 \mu$ beträgt, doch kann man auch hier und da Dimensionen bis zu 13-15 $\mu$ messen.

Zerzupfungspräparate ergaben für die hintere Wand folgende Zahlen.

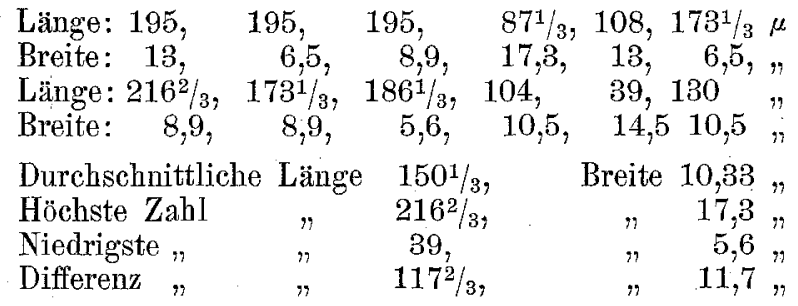

Es ist zu diesen Zahlen noch zu bemerken, dass die beiden Muskelzellen, welche 17,3 und 14,5 in die Breite messen, ein eigenthümlich glasiges Aussehen hatten; ihre Kerne waren ohne Essigsäurezusatz undeutlich zu sehen, sahen geschwollen aus und machten ebenfalls einen glasartigen Eindruck. Diese Zellen, denen ähnliche übrigens in anderen Gesichtsfeldern noch mehrere gemessen wurden, waren im Verhältniss zu ihrer Länge auffallend dick. Sie gleichen ganz demjenigen, welche Paul Dittrichs in der Zeitschrift für Heilkunde 1889, Bd. X. p. 82, als hyalin degenerirte beschreibt und Tafel I. abbildet.

Schlösse man diese von den Messungen aus, so würden sich die Durchschnittsmaasse auf Länge: 167,6 $\mu$, Breite: $9,2 \mu$ stellen. 
Die mikroskopische Untersuchung der vorderen Wand ergiebt, dass es sich auch hier um eine vorwiegend muskuläre Hypertrophie handelt. Von einer Schichtung ist aber hier auch mikroskopisch nichts zu sehen. Schnitte, welche in verschiedenen Richtungen augelegt waren, ergaben, dass die Muskelbündel filzartig ineinander verflochten waren. Zwischen ihnen befindet sich Bindegewebe, welches zum kleineren Theil kernarm glänzend, zum grösseren Theil kernreicher und aufgelockert ist. Nur dicht an der Schleimhaut sieht man etwas kleinzellige Infiltration. Innerhalb der Muskelbündel ist das Bindegewebe hier und da vermehrt. Die Muskelbündel sind breit und enthalten stark vergrösserte Muskelzellen. Die Durchschnittsgrösse scheint nach Messungen an Querschnitten in einzelnen Muskelbündeln $8-9 \mu \mathrm{zu}$ betragen, in anderen scheint keine Hypertrophie vorzuliegen. Zerzupfungspräparate ergaben folgendes Resultat:

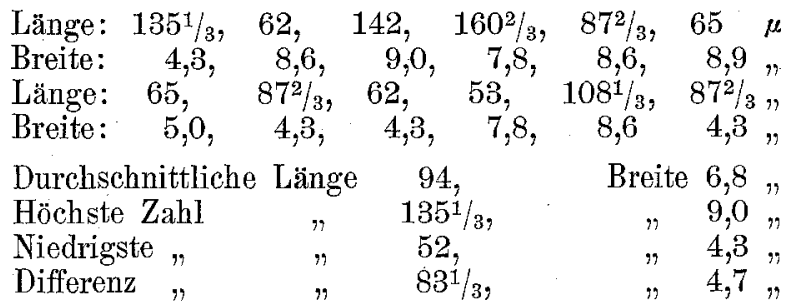

19. Uterus. Von einer 48 jährigen Frau.

Der Rest des durch supravaginale Amputation entfernten Uterus ist $16 \mathrm{~cm}$ lang, die Wanddicke beträgt $21 / 2 \mathrm{~cm}$, die Uterushöhle wird durch ein $9 \mathrm{~cm}$ im Durchmesser haltendes sehr hartes, viel Bindegewebe enthaltendes submuköses, breitbasig aufsitzendes Fibromyom ausgefüllt. Die Wand sah weissröthlich aus, zeigte keine Schichtung und fühlte sich hart an. Die mikroskopische Untersuchung ergiebt, dass Bindegewebe und Muskulatur gleichmässig an der Bildung der Wand betheiligt sind, das Bindegewebe ist zellarm, faserig. An einigen Stellen wiegt die Muskulatur vor, doch kann man Muskelzellen bis zu 8-9 $\mu$ Durchmesser messen. Es liegt aber weder eine allgemeine noch eine vorwiegende Vergrösserung der Muskelzellen vor.

Zerzupfungspräparate ergaben folgendes Resultat:

$\begin{array}{lccccc}\text { Länge: } 43, \quad 52, & 35^{1} / 3, & 63^{1} / 3, & 77, & 45 & \mu \\ \text { Breite: } 4,3, \quad 6,15, & 4,3, & 3,9, & 4,5, & 5,0 \% \\ \text { Länge: } 82^{2} / 3, \quad 40, & 481 / 3, & 92, & 73, & 52^{2} / 3 " \\ \text { Breite: } 8,6, \quad 6,5, & 4,2, \quad 8,9, & 5,8, & 9,0 \% \\ \text { Durchschnittliche Länge } & 58^{1} / 2, & \text { Breite } & 5,96, \\ \text { Höchste Zahl, Länge } & 92, & " & 9,0 \% \\ \text { Niedrigste Zahl " } & 35^{2} / 3, & " & 3,9 " \\ \text { Differenz } & " & 56^{1} / 3, & " & 5,1 "\end{array}$

\section{Uterus. Von einer 44 jährigen Frau.}

Es stand mir ein Theil der Wand zur Verfügung, die Aufzeichnungen des pathologischen Instituts ergaben, dass es sich um einen Uterus gehandelt habe, in dessen Höhle ein polypös-submuköses Myom von bedeutender Grösse hineingeragt habe und in dessen Wand sich noch viele kleine interstitielle und submuköse Myome befunden hätten. Das vorliegende Stück zeigt nicht sehr ausgeprägte aber deutliche Schichtung. 
Die mikroskopische Untersuchung ergiebt, dass eine reich entwickelte Muskulatur zwischen lockerem, schon faserigem aber noch ziemlich zellreichen Bindegewebe liegt, welches den Eindruck macht, als sei es stark serös durchtränkt gewesen. In demselben verlaufen viele weite Gefässe. Entzündliche Veränderungen werden nicht bemerkt. Die einzelnen Muskelzellen sind durchweg vergrössert. Es werden Muskelzellen gemessen, deren Durchmesser $19^{1 / 2} \mu$ beträgt, durchschnittlich gehen die Durchmesser aber nicht über $13 \mu$ hinaus. Von den grösseren über $13 \mu$ hinausmessenden Muskelzellen unterscheiden sich viele in den mit Pikrolithionkarmin gefärbten Präparaten von den normalen stark gelb mit rothem Kern gefärbten Muskelzellen durch die mangelnde Färbung ihres Zellleibes. Derselbe erscheint blassgelb oder bis auf die äussere Umgrenzung geschwunden. Der Kern ist dabei aber sehr wohl gefärbt und sieht unverändert aus. Diese Zellen haben grosse Aehnlichkeit mit quergetroffenen Nervenfasern, wobei der Kern dem Axencylinder ähnlich ist. In den Zerzupfungspräparaten konnte trotz dahin gerichteter Bemühungen nichts diesen Zellen entsprechendes isolirt werden, weshalb ich mich vorläufig auf die obige Beschreibung beschränken muss. Das Nicht-Erscheinen dieser Zellen in Zerzupfungspräparaten lässt auf eine leichte Zerstörbarkeit derselben schliessen. Die erhaltenen Zahlen sind folgende:

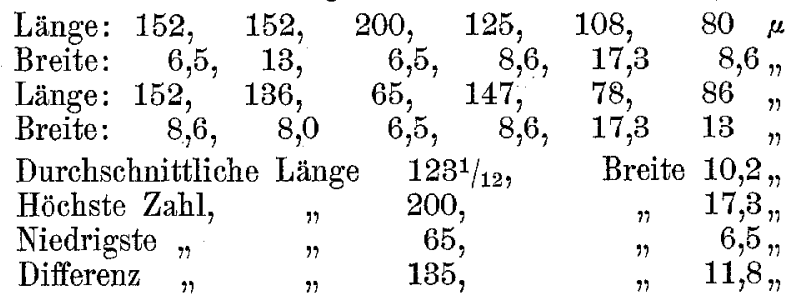

21. Uterus. Von einer 51 jährigen Jungfrau.

Dieser Uterus war $18 \mathrm{~cm}$ lang, sein Cavum wurde von einem kindskopfgrossen, $15 \mathrm{~cm}$ Durchmesser haltenden submukösen Fibromyom bis zum äusseren Muttermunde hin ausgefüllt. Das Fibromyom inserirt sich an der Hinterwand in der Nähe des Fundusscheitels. In der hinteren Portiolippe befindet sich noch ein hühnereigrosses interstitielles Fibromyom. Die Wände sind $2^{1 / 2} \mathrm{~cm}$ dick, zeigen deutliche Schichtung und fühlen sich weich an. Mikroskopisch besteht die Wand aus hypertrophischer Muskulatur; welche von ziemlich reichlichem, aber lockerem, von vielen weiten Gefässen durchsetztem Bindegewebe umgeben ist. Im Bindegewebe liegen vereinzelte Mastzellen und hier und da Häufchen von Rundzellen. Nur an einer Stelle sieht man die letzteren in grosser Anzahl. Die Muskelzellkerne sind lang und haben feines Chromatingerüst mit deutlich trennbaren Fäden, die einzelnen Muskelzellen sind recht gross, sie messen in einzelnen Fällen auf dem Querschnitt 171/3 bis $21^{2} / 3 \mu$ in der Breite, im Allgemeinen scheint der Durchmesser $10 \%$ zu betragen. Zellen wie die beim vorigen Fall beschriebenen, deren Leib schwach, deren Kern stark gefärbt ist, kommen auch, aber nur vereinzelt, vor. Isolirte Muskelzellen hatten folgende Maasse:

$\begin{array}{lccccccc}\text { Länge: } & 130 & 169 & 108^{1 / 3} & 262 & 108{ }^{1 / 3} & 195 & \mu \\ \text { Breite: } & 8,6 & 7,8 & 13 & 6,5 & 10,9 & 13 & " \prime \\ \text { Länge: } & 152 & 209 & 209 & 186 & 86^{2} / 3 & 179 & n \\ \text { Breite: } & 13 & 13 & 9,36 & 17,18 & 15,6 & 6,5 \%\end{array}$




\begin{tabular}{|c|c|c|c|c|}
\hline Durchschnittliche & änge & $166^{5} / 36 \mu$, & Breite & 112 \\
\hline Höchste Zahl & 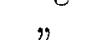 & 262 & $"$ & 17,18 \\
\hline Niedrigste Zahl & & $86^{2} / 3$ & & 6,5 \\
\hline Differenz & & $175^{1 / 3}$ & & 10,68 \\
\hline
\end{tabular}

22. Uterus von einer 42 jährigen Frau.

Das Organ misst $19 \mathrm{~cm}$ in der Länge; ein kindskopfgrosses Myom von $13 \mathrm{~cm}$ Durchmesser füllt das Uteruscavum; der Cervixcanal ist ganz erweitert, die Uteruswand ist $3 \mathrm{~cm}$ dick und weich. Sie zeigt eine Schichtung ihres Gewebes. Leider war es nicht möglich, bei den Sehnittpräparaten, da der Uterus schon lange aufbewahrt wird, gute Kernfärbungen zu erzielen; doch lässt sich noch feststellen, dass die Wand zum grossen Theil aus Muskulatur besteht, zwischen welcher sich ein lockeres, von weiten Gefässen durchsetztes Bindegewebe befindet. Es konnten in grösserer. Anzahl Muskelzellen gemessen werden, deren Breitendurchmesser bis zu $13 \mu$ betrug.

Zerzupfungspräparate ergaben folgende Zahlen:

\begin{tabular}{|c|c|c|c|c|c|}
\hline änge: & 140 & 87 & 82 & 74 & 100 \\
\hline 8,6 & 8,6 & 6,5 & 4,3 & 4,3 & 4,3 \\
\hline $00^{\prime}$ & 65 & 65 & $108^{1 / 3}$ & 173 & $3^{1 / 3}$ \\
\hline Breite: & 8,9 & 13,0 & 8,6 & 13,0 & , \\
\hline chnittlic & Länge & $101^{1 / 3}$ & ${ }_{3} \mu$ & Breite & 8,53 \\
\hline & & 173 & & $"$ & 17,3 \\
\hline 年 & & 61 & & $"$ & 4,3 \\
\hline ifferen & & 112 & & " & 13,0 \\
\hline
\end{tabular}

23. Uterus von einer 38 jährigen Frau, welche-im 21. Lebensjahre eine schwere Geburt durchmachte, darauf fünfmal einen Abort hatte, wobei immer heftige und langdauernde Blutungen auftraten.

Vom 27. bis 38. Jahre war sie fortwährend krank und schwach. In der letzten Zeit ist kein Abort in der Krankengeschichte erwähnt, dagegen traten Schmerzen beim Stuhlgang ein. Die Schmerzen nahmen zuletzt einen wehenartigen Character an.

Das vorliegende Organ misst $12 \mathrm{~cm}$ in der Länge, trägt im Fundus ein gänseeigrosses submuköses Myom; seine Wände sind $1 / 3 \mathrm{~cm}$ dick. An der Aussenseite des Fundus befinden sich noch zwei subseröse FibroMyome. Der Cervixcanal ist nicht erweitert, sondern eng und wurstförmig verlängert; die Cervix misst $4 \mathrm{~cm}$ in der Länge. Auf dem Durehschnitt der Uteruswand erkennt man eine ziemlich undeutliche Schichtung. Man sieht mit dem Mikroskop, dass die mittleren Schichten der Uteruswand ziemlich viel Bindegewebe enthalten, besonders in der Nähe der hier verlaufenden grösseren Gefässe. In der Nachbarschaft derselben sieht man grosse Haufen ein- und mehrkerniger Leukocyten. Auch sind hier und da einzelne Mastzellen im Bindegewebe zerstreut. Nach innen und nach aussen von dieser mittleren Schicht kommt man auf Muskelbündel, die von verhältnissmässig geringen Mengen Bindegewebes umschlossen sind. In diesem sind die Muskelzellen mächtig hypertrophisch. Man kann auf Querschnitten Durchmesser von 13, 171/3 $-21^{2 / 3} \mu$ messen. Die letzten aber meistens bei solchen Muskelfaserzellen, die beim Fall 20 schon beschrieben worden sind, deren Leib blass und ungefärbt erschien, 
während der Kern sehr gut gefärbt war. Manchmal sahen in diesem Falle die Kerne blasig aufgetrieben aus. Sie haben dann auch verwaschene Grenzen gegen die Umgebung. Es sind hier ganze Muskelbüudel von dieser Veränderung betroffen. An einzelnen Stellen sah ich auch Muskelzellen, deren Leiber für die bindegewebige Umhüllung zu klein geworden waren; dies war manchmal in solchem Grade der Fall, dass es nicht durch Schrumpfungsvorgänge allein bei der Härtung erklärt werden konnte. Manchmal scheint nur noch der Kern vorhanden zu sein, der frei in der Bindegewebsalveole liegt.

Im puerperalen Uterus sah ich ähnliche Verhältnisse, auf deren Erklärung: ich später zurückkommen werde.

An Zerzupfungspräparaten wurden keine der bei Fall 20 beschriebenen Muskelzellen gefunden.

Messungen an Zerzupfungspräparaten:

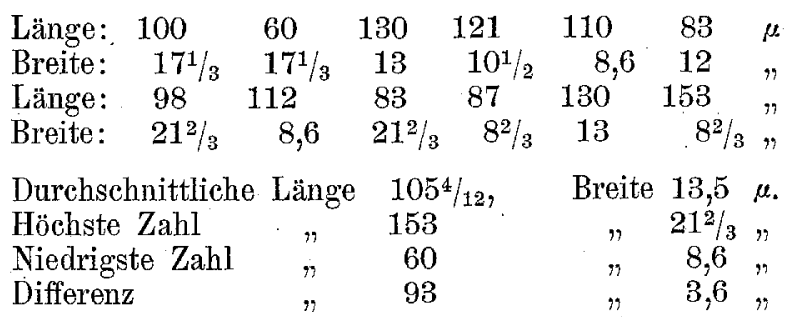

Den vorliegenden Fall, bei dem trotz nicht verdickter Uteruswand starke Hypertrophie der Muskelzellen gefunden wird, erkläre ich mir folgendermaassen. Durch die schweren entzündlichen Veränderungen, deren lange Dauer durch die fünf Aborte, deren Fortbestand durch die Anwesenheit grösserer Mengen gelapptkerniger Leucocyten erwiesen wird, ist ein Theil des Uterusgewebes zu Grunde gegangen. Es macht auch factisch den Eindruck, als sei die Zahl der Muskelbündelchen geringer denn sonst.

Durch die Entwickelung des Myoms wurden nun die Muskelzellen zur Hypertrophie gereizt, wegen ihrer geringen Zahl konnten sie aber nur die vorher verdünnte Uteruswand auf ihre normale Dicke bringen. Neubildungen fanden, wenn man solche überhaupt zulassen will, bei der sehr anämischen Patientin nicht statt.

24. Uterus.

Dieses Organ wurde von einem 18 jährigen Mädchen genommen, welches an Atresie der Vagina litt. Der Verschluss befand sich an der Grenze zwischen der unteren und oberen Hälfte der Vagina. Mit Beginn der Menstruation hatte sich eine Hämatometra ausgebildet und die daraus entstehenden Beschwerden veranlassten eine Operation, welche am 5. 4.1895 stattfand.

Auf die Operation hin verkleinerte sich der Uterus, wurde aber nicht normal gross. Leider trat Infection ein, es floss seit 
dem 16. 4. Eiter aus, es sammelte sich Eiter: im Uterusraum und in den Tuben. Am 21.4. erlag die Patientin allgemeiner Peritonitis.

Die Verhältnisse sind folgende: Die Innenfläche des unteren Theils der Scheide ist gelblich gefärbt und auffallend dünn, der obere Theil der Scheidenschleimhaut in einer Ausdehnung von $3 \mathrm{~cm}$ dunkelbraum gefärbt, die Fläche ist hier höckrig und fühlt sich derb an; die Dicke der Wand beträgt $3 \mathrm{~cm}$. Das Innenlumen der Vagina hat an dieser Stelle $10 \mathrm{~cm}$ Umfang. Der Uterus selbst ist vergrössert. Die Innenhöhe der Uterushöhle beträgt $6^{1} / 2 \mathrm{~cm}$, die Innenbreite am Fundus $4 \mathrm{~cm}$. Die Wände zeigen starke Hypertrophie, besonders an der Portio cervicalis, dort beträ̈gt die Wanddieke $2 \mathrm{~cm}$, am Fundus $1 \frac{1}{2} \mathrm{~cm}$. Die Uterusschleimhaut ist schwarzroth und stark gewulstet. Die Uteruswand sieht auf dem Durchschnitt weiss-röthlich aus und zeigt eine feine Schichtung. Bei mikroskopischer Untersuchung sieht man, dass die Schleimhaut stark verändert ist; sie ist sehr reichlich von Blutgefässen durchzogen. Im ovalzelligen, theilweise der Kernfärbung entbehrenden Stroma sind sehr wenig Drüsen übrig geblieben; die vorhandenen haben entweder gar kein oder ganz nekrotisches Epithel. In der Nähe der Schleimhaut finden sich kleinere, mehr der Mitte zu grössere, dem stark verdickten serösen Ueberzug zu wieder kleinere Muskelbündelchen, welche von ziemlich Iockerem Bindegewebe umgeben sind. Im Bindegewebe und um die Gefässe herum sieht man hier und da kleinzellige Infiltration; in Lymphräumen und Gewebsspalten Haufen und Züge ron Mikrokokken. In dem bindegewebigen Maschenwerk, dessen Maschen $5-8-9 \mu$ Durchmesser haben, liegt in jeder Masche eine, entweder dieselbe ganz oder nur theilweise ausfüllende Muskelzelle. Manchmal sind mit gut gefärbtem. Kern versehene Muskelzellen ganz bedeutend kleiner als die Maschen; sie liegen dann in deren Mitte, und zwischen ihnen und der Innenwand der Bindegewebsmasche ist ein völlig freier Raum. Oft ist die Muskelsubstanz um den Kern so gering, dass man fast nichts mehr von ihr sieht; der Kern ist aber trotzdem gut gefärbt und ohne Besonderbeiten. An anderen Stellen liegt um den manchmal intumescirten Kern herum eine die Masche ganz ausfüllende, eigenthümlich glasig, helle Substanz, sodass wieder das Bild des Nervendurchschnitts entsteht.

Dieser Verschiedenheit der Muskelzelldurchschnitte entsprechend giebt auch das Resultat bei Zerzupfungspräparaten Ausdruck.

\begin{tabular}{|c|c|c|c|c|c|c|c|}
\hline änge: & $34^{1} / 3$ & $821 / 3$ & $34^{2 / 3}$ & $86^{2} / 3$ & 65 & & $\mu$. \\
\hline Breite: & $6^{1 / 5}$ & 6,5 & 5,0 & 6,5 & 6,8 & 7,02 & \\
\hline Länge: & & 65 & 52 & $55^{\prime}$ & 52 & 26 & $"$ \\
\hline Breite: & 4,3 & 8,6 & 8,0 & 5,0 & 5,46 & 5,0 & $n$ \\
\hline Länge: & $30^{1 / 3}$ & 43 & $34^{1 / 3}$ & $21^{2 / 3}$ & 52 & 45 & \\
\hline Breite: & 4,3 & 3,0 & 6,24 & 3,9 & 6,5 & 7,8 & $5,0 \mu$. \\
\hline \multicolumn{3}{|c|}{ Durchschnittliche Länge } & \multicolumn{2}{|l|}{49,1} & Breite & \multicolumn{2}{|l|}{5,56} \\
\hline \multicolumn{2}{|c|}{ Höchste Zahl } & $n$ & $86^{2} / 3$ & & $n$ & 8,6 & \\
\hline \multirow{2}{*}{\multicolumn{2}{|c|}{$\begin{array}{l}\text { Niedrigste Zahl } \\
\text { Differenz }\end{array}$}} & $"$ & $21^{2} / 3$ & & $n$ & 3,0 & $n$ \\
\hline & & & & & & & \\
\hline
\end{tabular}

Bei der Besprechnng der 12 letzten Fälle möchte ich den Fall 16 vorweg nehmen. Es handelt sich hier um eine Veränderung der Wand, welche mit den sonst gefundenen nichts gemein 
hat und auch sich an keine sonstige krankhafte oder physiologische Form, die mir bekannt ist, anlehnt.

Die zerstreut liegenden hypertrophischen Muskelkomplexe könnten als beginnende allgemeine Hypertrophie aufgefasst werden, wenn es sich nicht zum grossen Theil um Muskelbündelchen handelte, welche entweder in der Nähe von gefässartigen Räumen sich befinden oder unmittelbar mit ihnen zusammenhängen. Es ist mir sehr wahrscheinlich, dass diese gefässartigen Hohlräume stark veränderte Venen sind. In der Wand eines von käsiger Uretritis ergriffenen Ureter, sah ich ein Gefäss, welches ähnlich wie diese Hohlräume von gleich ihm quergetroffenen also längsverlaufenden, ausserhalb der unveränderten Media liegenden Muskelbündeln umgeben war.

Bei den übrigen Fällen von einfachen interstitiellen Myomen zeigten sich entweder, trotz der manchmal kolossalen Vergrösserung des Uterus entweder keine oder nur sehr geringe Vergrösserungen der Muskelzellen, bis auf den Fall 18. Bei diesem tritt uns in der hinteren Wand, welche das Myom enthält, Schichtung der Substanz und sehr bedeutende Vergrösserung der Muskelzellen entgegen; während die vordere ungeschichtete Wand Muskelzellen von etwas geringerer Grösse trägt. Wir haben es hier mit einem Fall zu thun, bei dem die Uterushöhle verhältnissmässig wenig in Mitleidenschaft gezogen worden ist. Es kann also auch von einer Auslösung der immanenten Anlage oder von Arbeitshypertrophie nicht gut die Rede sein, wenigstens nicht in dem Sinne, dass wir diese Leiden als durch abnormen Inhalt der Uterushöhle zu Stande gebracht uns denken. Es wäre also hier eine Ausnahme von der Regel festzustellen. Eine gleiche bildet unter den submucösen Myomen der Uterus Nr. 19, bei welchem sich, trotzdem es sich um ein submucöses Myom, also um eine Ausfüllung des Uterusraumes durch einen Fremdkörper handelte, keine bedeutende Hypertrophie der Muskelzellen nachweisen liess. Leider liess sich durch die Krankengeschichte nichts genaueres über die Entstehung der Tumors nachweisen. Es könnte sich nach den Arbeiten von Tillaux und Labbé auch um die nachträgliche Entstehung eines Fibro-Myoms in einem idiopathisch hypertrophirten Uterus handeln. Dafür liess sich aber kein Anhaltspunkt finden. Die unter Fall 20, 21 and 23 beschriebenen quergetroffenen Nervenfasern ähnliche Muskelzellen stellen eine Degenerationserscheinung besonderer Art dar. 
Vielleicht handelt es sich hier um Fäulnisserscheinungen. Es bleiben noch die Muskelzellen von den Fällen Nr. 20, 23 und 24 zu erwähnen, deren Grösse in einem auffälligen Missverhältniss zur Weite ihrer bindegewebigen Scheide steht.

Es wurden derartige Muskelzellen von mir auch in Schnitten aus zwei puerperalen Gebärmüttern gesehen, welche atonisch waren. In einem dritten Falle war die Patientin ungefähr 14 Tage nach Abort im 5. Schwangerschaftsmonat an Peritonitis zu Grunde gegangen. Blutungen stellten sich drei Wochen vor dem Tode ein. Die letzten Abortfetzen wurden acht Tage vor dem Tode ausgeräumt; der Uterus war gut contrahirt aber noch mannsfaustgross. In diesem Falle zeigten sich solche Bilder nicht. Die Muskelzellen waren nur etwas vergrössert, ihre Maasse betrugen zwisehen $43-86$ in der Länge, $4^{1} / 2-8,6$ in der Breite.

Bei den beiden ersten Gebärmüttern, deren Trägerinnen 2 und 4 Tage nach der Geburt gestorben waren, fanden sich theils Muskelzellen, welche normal gross waren, theils solche von über 200 Länge und 17-20 Breite. An Schnittpräparaten ist dies Verhältniss so, dass neben stark gefärbten, manchmal kernlosen, gequollen aussehenden Muskelfasern, solche in ihren nicht kollabirten Bindegewebsscheiden liegen, bei denen manchmal nur ein wenig Protoplasma, manchmal scheinbar nur der Kern, welcher gut gefärbt ist, übrig geblieben ist. Ich führe hierzu eine Beobachtung. Sänger's an; dieser untersuchte das schwangere rudimentäre Horn eines Uterus bicornis, welches 9 Wochen nach dem Tode der bis in den 7. Mondmonat gediehenen Frucht amputirt worden war. Dem Fruchttode war eine 12 stündige Wehenthätigkeit voraufgegangen, das Uterushorn hatte sich in den folgenden Wochen wesentlich verkleinert. Die Frucht war aber nicht ausgestossen worden. Trotzdem hatten sich die Muskelzellen soweit zurückgebildet, wie es am Ende der Involutionsperiode geschehen zu sein pflegt. Es kạn also eine Rückbildung der Muskelzellen stattfinden, ohne dass der Uterus seine normale Grösse -wieder erreicht hat. In den von mir untersuchten atonischen Gebärmüttern hatte nun anch die Wand $2^{x} / 2 \mathrm{~cm}$ Dicke und fühlte sich schwammig an, sie war sehr succulent und blutreich. Auch in diesem, wie Sänger nachgewiesen hat, erfolgt sofort nach der Geburt eine Rückbildung der Muskelzellen. Der Uterus aber zieht sich wegen der Atonie nicht zusammen; die klaffenden Bindegewebsspalten werden durch das in Folge der Metritis und Endometritis puerperalis reichlich 
vorhandene Exsudat ausgefüllt; so haben wir vielleicht eine Erklärung der oben beschriebenen Bilder. Die Muskelzellen verkleinern sich, die Bindegewebsscheiden werden nicht zusammengezogen. Die verkleinerte Muskelzelle bleibt also frei liegen in weitem Umkreise von Bindegewebe umgeben.

Das gleiche kann in einem Uterus eintreten, der in Folge eines in seiner Höhlung gelegenen Fremdkörpers hypertrophisch geworden war. Der Fremdkörper kann sich so verändern, dass er ähnliche Einflüsse wie die abgestorbene Frucht auf den Uterus ausübt. Starb die Frucht ab, so verkleinerten sich in dem Sänger'schen Falle die Muskelzellen. Geht der abnorme Inhalt der Uterushöhle bei unseren Fällen eine ähnlich wirkende Veränderung ein, so wird der Erfolg ebenfalls Verkleinerung der Muskelzellen sein. Da die Uterushöhle sich aber hier nicht entleert, kann das bindegewebige Gerüst der Verkleinerung der Muskelzellen nicht folgen. Es müssen ähnliche Bilder wie bei den beschriebenen atomischen Gebärmüttern entstehen.

Es ist dieses nur eine Hypothese; um sie zu beweisen, müsste nachgewiesen werden, dass bei bestimmter Veränderung des abnormen Inhalts stets dieselben Erscheinungen auftreten. Aehnliche Verhältnisse finden wir bei der zur Pyometra gewordenen Hämatometra. Auch dort erscheinen in den Zerzupfungspräparaten die Muskelzellen zum Theil viel kleiner, als sie wabrscheinlich ursprünglich gewesen sind, die einen füllen ihre Hülle noch aus, den andern ist sie viel zu weit geworden. Die jetzige Weite der Bindegewebshülle stellt noch nicht ganz die ehemalige Breite der Muskelzellen dar, denn nach der Punction hat sich der Uterus stark verkleinert, wenn auch nicht auf sein normales Volumen. Vielleicht hat damals die Rückbildung der Muskelzellen eingesetzt, vielleicht erst als der Rest des die Uterushöhle noch füllenden Blutes verjauchte.

Zum Schluss möchte ich noch eine Zusammenstellung von den von Sänger in schwangeren und puerperalen Gebärmüttern und von mir in meinen Fällen der pathologischen Vergrösserung gefundenen Maasse der Muskelzellen geben. Ich führe hier nur Messungen aus den Organen an, bei welchen annähernd gleichmässige Verhältnisse im ganzen Uterus vorlagen, lasse also Nr. 4 bis 7 und Nr. 16 fort. Bei Nr. 8 konnten keine Messungen gemacht werden. 


\section{Sänger's Messungen.}

\section{Länge:}

Uter. gravid. $\left\{\begin{array}{cc}\text { VImens. } & 244,1 \\ \text { VIII }, & 172,6\end{array}\right.$

Uterus 4 Std. p. part.

$\begin{array}{lrlll}n & 6 & n & n & n \\ n & 3 & \operatorname{Tage} & n & n \\ n & 4 & n & n & n \\ n & 8 & n & n & n \\ n & 9 & n & n & n \\ n & 13 & n & n & n \\ & 16 & n & n & n \\ & 35 & n & n & n \\ n & 55 & & & n\end{array}$

Meine Messungen.

Länge:

Uterus 21

" 18

" 12

" 20

$n \quad 22$

$" 23$

$" 9$

" 11

$" 19$

" 15

" 17

" 3

$" 24$

" 14

" 10

, 1

" 13

n. 2 $\left.\begin{array}{l}145,1 \\ 171,6\end{array}\right\} 158,3$ $\left.\begin{array}{l}122,2 \\ 112,5\end{array}\right\} 117,4$ $\left.\begin{array}{l}95,9 \\ 69,4\end{array}\right\} 82,7$

64,4

$\left.\begin{array}{l}33,5 \\ 31,9\end{array}\right\} 32,7$

23,4

$\left.\begin{array}{l}25,4 \\ 17,4\end{array}\right\} 21,2$

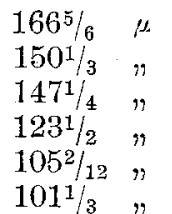

$971 / 10 \%$

74,4

$571 / 2 "$

$52^{2} / 3 "$

$44^{1 / 2} "$

$45^{11 / 18} "$

$491 / 10 "$

$491 / 4$

$421 / 36 n$

$415 / 12 "$

$40^{1 / 3}$ "

14,5 "
Breite:

$\left.\begin{array}{r}11,9 \\ 9,1\end{array}\right\} 10,5$

$\left.\begin{array}{l}13,6 \\ 11,5\end{array}\right\} 12,2$

$\left.\begin{array}{r}11,7 \\ 9,4\end{array}\right\} 10,5$

$\left.\begin{array}{l}9,2 \\ 6,7\end{array}\right\} 8,0$

6,7

$\left.\begin{array}{l}6,3 \\ 5,9\end{array}\right\} 6,1$

5,8

$\left.\begin{array}{l}6,2 \\ 4,7\end{array}\right\} 5,45$

Breite:

$11,5 \mu$

10,33 "

11,0 "

$10,2 "$

8,53,

13,5

8.1,

4,83

5,56

4,97,

$5,5 \quad$

4,4 "

5,56

5,93

4,26 "

4,08,

3,49 "

$3,1 \quad$ "

Uterus 1-3 Uteri mit Netritis.

$$
\begin{aligned}
& \text { " 4- } 7 \text { " } " \text { Carcinomen. } \\
& " \text { 8-9 " " } " \text { Sarkom und Deciduom. } \\
& " \text { 10-12 " } " \text { multiplen Myomen. } \\
& " 13-18 \quad " \quad \text { "interstitiellen " } \\
& \text { " 18-23 " " submukösen " } \\
& \text { "24 " " " Hämato-Pyometra. }
\end{aligned}
$$

Es fehlen in der obenstehenden Zusammenstellung No. 4-7, 8 und 16 aus oben angeführten Gründen. 
Es geht aus dieser Zusammenstellung hervor, dass, wenn sich Myome an oder in der Uteruswand bilden, Hypertrophie der Muskelzellen eine sehr häufige Begleiterscheinung ist. Beinahe regelmässig tritt dies bei den submueösen Myomen auf und bei den Erkrankungen, welche gleich diesen eine langsam wachsende Ausdehnung der Uterushöhle durch abnormen Inhalt veranlassen. Seltener kommt es bei solitären interstitiellen Myomen vor. Dass die Hypertrophie der Muskelzellen, wie sie sich bei multiplen Myomen fanden, theils dưreh einen allgemein fibro-myomatösen Zustand des ganzen Uterus sich erklären, theils auf dieselben Ursachen, wie sie bei den submucösen Myomen, Arbeitshypertrophien und Erregung der immanenten Anlage zurückzuführen sind, habe ich schon erwähnt. Der Vergleich mit den von Sänger gefundenen Zahlen für die Muskelzellen des puerperalen Uterus ergiebt, dass die von mir gefundenen Hypertrophien an die des Uterus puerperalis 4 Stunden nach der Guburt heranreichen.

Es ist durch die angeführten Fälle bewiesen, dass zweierlei Vorgänge im Myometrium Vergrösserung des Uterus bewirken können.

Dieselben sind Vermehrung der einzelnen Gewebselemente und Vergrösserung der Muskelzellen. Beide Vorgänge greifen manchmal in einander über und können zu gleicher Zeit vorhanden sein. Hyperplastische Vorgänge, Vermehrung des Bindegewebes und der Zahl der Muskelzellen finden wir hauptsächlich bei Metritis, Carcinomen und interstitiellen Myomen. Es wirkt hier in erster Linie ein Reiz auf das Myometrium ein, der weder eine erhöhte Arbeitsleistung des Organs veranlasst, noch die Bethätigung der dem Uterus eigenthümlicben Anlage anregt, sich bei der Schwangerschaft zu vergrössern.

Die normale Function des Uterus besteht in Vergrösserung, um der wachsenden Frucht Raum zu bieten, seine Muskulatur verstärkt sich, um dieselbe nach Ausreifung ausstossen zu können. Weder das Bestehen von Metritis noch die Anwesenheit von Carcinomen bedingen an und für sich, dass ähnliche Anforderungen, wie sie physiologischer. Weise gestellt werden, an das Organ herantreten und auch bei den interstitiellen Myomen scheint dies richt der Fall zu sein.

Der Uterus reagirt in Folge dessen bei diesen krankhaften Veränderungen nicht in der Weise, wie es normal seine eigenthümliche Anlage mit sich bringt, sondern durch Wucherungs- 
vorgänge, welche sowohl Vermehrung des Bindegewebes wie der Muskulatur bewirken. Ob sich mehr Bindegewebe oder mehr Muskulatur bildet, hängt von Nebenumständen wie Alter der Patientin, die Heftigkeit der entzündlichen Reize u. s. w. ab. Immer aber scheint das neugebildete Gewebe sich dadurch vom normalen zu unterscheiden; dass das Bindegewebe sowohl in der Nähe der Gefässe wie in den Muskelbündeln selbst eine mehr homogene, weniger faserige Beschaffenheit annimmt. Meistens ist auch in den neugebildeten oder verbreiterten Muskelbündeln das Bindegewebe vermehrt. Im späteren Stadium scheint durch das sich retrahirende Bindegewebe eine Verkleinerung der Muskelzellen einzutreten. Dass dort, wo frische Wucherungen statthaben, auch Vergrösserungen der einzelnen Elemente geringeren Grades vorkommen, ist nicht auffallend. Vergrösserungen der Muskelzellen etwas höheren Grades und Auflockerung der Uteruswand der Schwangerschaft entsprechend, kommen in unregelmässiger Weise auch bei Carcinomen vor. Wie oben angeführt, lässt sich das darauf zurückführen, dass der Tumor den Cervicalkanal verlegte und dadurch die Ausstossung des Inhaltes der Uterushöhle erschwerte. Interstitielle Myome müssen in ähnlicher Weise wie Metritis und Carcinome wirken, so lange sie noch klein sind; werden sie grösser, so ist es auffallend, dass die von ihnen bewirkten Veränderungen nicht häufiger den durch submucöse veranlassten entsprechen, auch wenn der Tumor bei seiner Vergrösserung allmählich die ihn von der Uterushöhle trennende Muskelschicht vortreibt, verdünnt und schliesslich in die Uterushöhle nur noch von wenig Muskelsubstanz überzogen hineinragt. Ich möchte es wagen, hierfür vorläufig eine Hypothese aufzustellen. Der in der Uteruswand liegende, sich entwickelnde Tumor kann in seinen Anfängen, da er mit der Uterushöhle nichts zu thun hat, nicht als Nachahmung des physiologischen Vorganges in der Schwangerschaft. wirken und Hypertrophie des Organs anregen. Er wird vielmehr wie jeder andere Tumor einen Fremdkörperreiz auf seine Umgebung ausüben und Wucherungsvorgänge veranlassen.

Diese werden als Endresultat Vermehrung der Muskulatur und des Bindegewebes, aber nicht, wie oben auseinandergesetzt, auch Bildung völlig normalen Gewebes haben. Hiermit ist ein Zustand geschaffen, der, wenn schliesslich das Myom in die Uterushöhle hineinragt, das Eintreten der echten Hypertrophie zwar nicht hindern, aber hemmen können wird. Tritt die Vergrösserung der 
vermehrten Muskelzellen schliesslich doch ein, so wird die Vergrösserung des ganzen Uterus bei interstitiellen Myomen natürlich eine ganz bedeutende sein, was mit den Beobachtungen Virchow's und Anderer gut übereinstimmt.

Es lassen sich durch diese Hypothese die Befunde gut erklären, namentlich spricht für sie, dass bei kleineren interstitiellen Myomen wie Nr. 13 neben leichter entzündlichen Veränderungen bedeutende Wucherungsvorgänge besonders im Bindegewebe gefunden wurden, während diese bei den grösseren Tumoren fast ganz fehlten und durch beginnende Vergrösserung der einzelnen Muskelzellen ersetzt wurde.

Es ist im Vorhergehenden schon wiederholt gestreift worden, dass in Folge krankhafter Veränderungen am Uterus Erregungen zur Einwirkung gelangen können, welche Aehnlichkeit haben mit denjenigen, welche physiologisch den Uterus zur Vergrösserung veranlassen.

Wächst in die Uterushöhle langsam ein Tumor ein, oder vermehrt sich ganz allmählich in seinem Cavum ein abnormer Inhalt wie Blut u. s. w., so stellt dies eine ziemlich genaue Nachahmung des Vorganges dar, der bei der Sehwangerschaft in dem langsamen Wachsthum der Frucht sich abspielt und wie die vorstehenden Untersuchungen ergeben, reagirt der Uterus in der That bei submucösen Myomen, Deciduomen und Sarcomen, die in sein Cavum einwachsen, and bei Hämatometra ähnlich wie bei Schwangerschaft. Sein Gewebe lockert sich auf, schichtet sich, seine Muskelzellen gehen eine wahre Hypertrophie ein. Dass daneben Wucherungsvorgänge, entzündliche Veränderungen mehr oder weniger, je nach der Art des Uterusinhalts, beim höchst malignen Deciduom mehr, wie beim gutartigen Myom mit ins Spiel kommen, ist natürlich. Den Hauptvorgang bildet aber doch die. Vergrösserung der Muskelzellen.

Ausser der Auslösung der immanenten Anlage zur Schwangerschaftsvergrösserung wirkt aber noch ein zweites Moment mit, welches zwar zu der Function des Uterus in naher Beziehung steht, aber eine weniger getreue Nachahmung der physiologischen Vorgänge veranlasst. Es finden nämlich bei abnormem Inhalt des Uterus Contractionen statt und auch diese scheinen geeignet zu sein, echte Hypertrophie hervorzurufen. Es entspricht dies wie erwähnt nicht den bei der Schwangerschaft eintretenden Verhält- 
nissen, wo der Uterus, nachdem er sich seines Inhaltes entledigt, sein Volumen durch dauernde Contractionen erheblich verkleinert. Seine Gefässe werden verengt, seine Muskelzellen verkleinern sich continuirlich, wie Sänger nachgewiesen hat.

In unseren Fällen sind die, Contractionen erstens nicht von der Heftigkeit, wie bei der Geburt, so dass ron einer sie schwächenden excessiven Arbeitsleistung, welche man als Grund für die Verkleinerung der Muskelzellen nach der Geburt in's Feld geführt hat, nicht gut die Rede sein kann. Sodann verkleinert der Uterus nie sein Volumen, eine Verengerung der Gefässe für die Dauer tritt nicht ein, die Contractionen bleiben rhythmisch, werden nie krampfhaft. Es ist höchst wahrscheinlich, dass diese Arbeitsleistung, wie bei anderen Organen mit glatter Muskulatur, zu einer Hypertrophie der Muskelzellen führt.

Fragt man sich wie im einzelnen die Erregung der immanenten Anlage des Organs zur Schwangerschaftshypertrophie durch langsam wachsenden Inhalt zu denken ist, so kann es sich um mechanische direct auf die Uteruswand einwirkende und nervöse Rejze handeln. Dass letztere bei der Sehwangerschaft mitwirken, beweist die Vergrösserung des Uterus bei Extra-Uterinschwangerschaft und die Vergrösserung des einen Horns bei Uterus bicornis, wenn das andere schwanger wird. Dass die auf nervösen Bahnen vermittelten Erregungen nicht allein ausreichen, das beweist das Zurückbleiben der Organe hinter der normalen Schwangerschaftsvergrösserung; Fibromyome scheinen eimen Einfluss auf nervösen Bahnen analog dem menschlichen Ei nicht auszuüben; denn bei subserösen FibroMyomen bleibt die Vergrösserung des Organs stets aus. Andererseits findet eine echte Uterushypertrophie auch dann statt, wenn von Nervenerregung nicht gut die Rede sein kann, bei Hämatometra; hier handelt es sich um einen sich stets vermehrenden abnormen Inhalt, der nicht organisirt ist und nicht in nervöser Verbindung mit dem Körper steht. Man könnte sagen, dass die nervösen Erregungen durch die Schleimhant vermittelt werden, doch ist unwahrscheinlich, dass diese, welche sich meist in degenerirtem Zustand befindet, die Erregungen zur Hypertrophie übermittelt.

Man kann also annehmen, dass bei Hämatometra und submucösen Myomen wesentlich mechanische Einflüsse die Hypertrophie veranlassen. Das gleiche findet bei polypösen Sarcomen und Deciduomen statt, bei den letzteren muss man allerdings die Frage 
offen lassen, ob es nicht die Decidua-ähnliche Wucherung ist, welche auf nervösem Wege die echte Hypertrophie bewirkt.

Es besteht also wahrscheinlich bei Metritis, Carcinomen und den meisten interstitiellen Myomen die Veränderung des Myometriums vornehmlich in einer Hyperplasie, bei langsam wachsendem abnormen Inhalt der Uterushöhle in der Regel in einer Auflockerung und Schichtung des Gewebes und Vergrösserung der Muskelzellen, die don Zuständen boi Schwangerschaft ähnlich ist.

Dieser Befund ist analog den von Herczel (Zeitschrift für klinische Medicin, Band 11, S. 32) und von A. Ritschl (Virchow's Archiv, Band 109) gemachten Beobachtungen. Herczel stenosirte experimentell Darmlumina und erhielt bei bedeutender Verstärkung der Darmwand reine Hypertrophie der glatten Muskelzellen, ohne Neubildung ron solchen. Ritschl stenosirte den Pylorus und hatte an der Magenmuskularis das gleiche Resultat.

Ausserdem setzte Ritschl Verletzungen und ätzte mit Crotonöl. Hierauf reagirten Uterus und Magen durch hyperplastische Vorgänge in der Muskelsubstanz und im Bindegewebe. Es entstand also bei Magen, Darm und Uterus bei erhöhten Anforderungen an die Function echte Hypertrophie der Muskelzellen, bei anderen Reizen hyperplastische Wucherung in Muskulatur und im Bindegewebe. Nach Th. Busachi (Ziegler's und Nauwerk's Beịträge, 1889, 4. Bd.) findet man bei künstlichen Darmstenosen, also bei functionell bedingter Hypertrophie neben starker Vergrösserung der Muskelzellen auch Kerntheilungen. Bei Verletzungen von Uterus und Darm sah er Kerntheilungen sowohl in der Muskulatur wie im Bindegewebe. Hierbei trat eine Vergrösserung der Muskelzellen ein. Doch betont auch Busachi für den ersteren Fall die Hypertrophie mehr als für die letzteren Fälle.

Diesen Beobachtungen schliesst sich das Resultat meiner Untersuchungen an. Dass die Erscheinungen mit der gleichen Ausschliesslichkeit und Regelmässigkeit zu Tage treten würden, wie beim Experiment, wo alle Fehlerquellen, besonders eine Combination von hauptsächlich inadäquaten Erregungen mit adäquaten wie beim Carcinom, oder hauptsächlich adäquaten mit inadäquaten wie beim Deciduom und anderen, vermieden werden können, ist bei pathologischen Veränderungen natürlich nicht zu erwarten. 
des Myometriums bei patholog. Vergrösserungen des Uterus etc. 219

Zieht man die das Gewebe durchtränkende Ernährungsfiüssigkeit in Betracht, so ist wahrscheinlich, dass bei erhöhten Anforderungen an die Function des Organs, durch vermehrte Blutzufuhr, mehr Nahrungsmaterial zugeführt wird, wobei sich die Zusammensetzung der Ernährungsflüssigkeit nicht wesentlich ändert. Bei Veränderungen anderer Art wird die Zusammensetzung der Ernährungsflüssigkeit eine Aenderung erfahren.

Auf beśsere Ernährung hin vergrössert sich also die Zelle vorzugsweise, auf Veränderungen in der Zusammensetzung der Ernährungsflüssigkeit geht sie mehr Kern- und Zellentheilungen ein. 